ITFA-2003-11

hep-th/0303006

\title{
A holographic reduction of Minkowski space-time
}

\author{
Jan de Boer ${ }^{1 *}$ and Sergey N. Solodukhin ${ }^{2 \dagger}$ \\ ${ }^{1}$ Instituut voor Theoretische Fysica, \\ Valckenierstraat 65, 1018XE Amsterdam, The Netherlands \\ ${ }^{2}$ Theoretische Physik, Ludwig-Maximilians Universität, \\ Theresienstrasse 37, D-80333, München, Germany
}

\begin{abstract}
Minkowski space can be sliced, outside the lightcone, in terms of Euclidean Antide Sitter and Lorentzian de Sitter slices. In this paper we investigate what happens when we apply holography to each slice separately. This yields a dual description living on two spheres, which can be interpreted as the boundary of the light cone. The infinite number of slices gives rise to a continuum family of operators on the two spheres for each separate bulk field. For a free field we explain how the Green's function and (trivial) S-matrix in Minkowski space can be reconstructed in terms of two-point functions of some putative conformal field theory on the two spheres. Based on this we propose a Minkowski/CFT correspondence which can also be applied to interacting fields. We comment on the interpretation of the conformal symmetry of the CFT, and on generalizations to curved space.
\end{abstract}

*jdeboer@science.uva.nl

${ }^{\dagger}$ soloduk@theorie.physik. uni-muenchen.de 


\section{Introduction}

Holography is a powerful principle, that after it had been proposed in [1] and [2] has found a concrete and quantitative realization in string theory in terms of the AdS/CFT correspondence [3, 4, 5]. Since then, many generalizations have been found, but all of these are deformations of, or limits of, the AdS/CFT correspondence. There have been attempts to generalize the AdS/CFT correspondence to de Sitter space (this was first discussed in [6] and [7]) but unfortunately string theory on de Sitter space, the dual field theory and the precise form of the correspondence are not well understood.

Besides Anti-de Sitter space and de Sitter space, which are solutions of the Einstein equations with negative and positive cosmological constant, there is a third class of spaces, namely those with zero cosmological constant. In particular, Minkowski space belongs to this class, and one may wonder what one can say about the existence and nature of a holographic dual description. There are local versions of entropy bounds [8] that also apply to Minkowski space, but these provide no specific insight about any dual description. An alternative approach is to take the decompactification limit of the AdS/CFT correspondence 9]. Though this is in principle a viable procedure, it has not led to an explicit dual description.

In this paper we will try something different. Minkowski space with metric

$$
d s^{2}=-d X_{0}^{2}+d X_{1}^{2}+\ldots+d X_{d+1}^{2}
$$

has three regions depending on whether $t^{2}<0, t^{2}=0$, or $t^{2}>0$, with

$$
-t^{2} \equiv X \cdot X=-X_{0}^{2}+X_{1}^{2}+\ldots+X_{d+1}^{2}
$$

The regions with $t^{2}>0$ and $t^{2}<0$ correspond to the inside and the outside of the light-cone respectively, whereas $t^{2}=0$ is the light-cone itself. For a fixed value of $t^{2}$, equation (1.2) describes a slice of Minkowski space whose geometry is either Euclidean anti-de Sitter, flat or Lorentzian de Sitter. The idea will be to apply holography to each slice separately, and in this way to effectively reduce the number of dimensions by one. All slices asymptote to the past or future light-cone, and therefore all dual field theories live on two d-spheres, which can be thought of as the boundaries of the past and future light-cone. It would appear as if we have lost two dimensions instead of one, but because of the infinite number of slices, we get an infinite number of fields on the two spheres, and this effectively reinstates one of the two lost dimensions. However, we cannot simply interpret the infinite number of fields as coming from a KK reduction of a theory in one dimension higher. The asymptotics of the metric (1.1) suggest that the extra dimension is a null dimension. Instead of trying to make sense of theories living on a space with a degenerate metric, we will mostly work directly with the infinite set of fields itself.

There are some obvious problems with this procedure. First of all, Minkowski space has de Sitter slices, and a holographic reduction along those slices requires us to apply the ill-understood dS/CFT correspondence. Luckily, for the purposes in this paper, we will only need some basic information like the form of two-point functions, and these are almost completely fixed by the symmetries of de Sitter space. Ultimately, if this whole procedure turns out to be consistent, one may try to reverse the logic and try to use known properties of Minkowski space in order to study holography for de Sitter space, but that is beyond the scope of the present paper. 
Another problem is the presence of the light-cone itself. Only non-interacting massless fields can spend their entire life inside the light-cone, but there are few if any of such degrees of freedom so it seems completely safe to leave the light-cone itself outside the discussion.

Despite these problems, we will find some encouraging results. For a free field we explain how the Green's function and S-matrix in Minkowski space can be reconstructed in terms of two-point functions of some putative conformal field theory on the two spheres. That this is at all possible is related to the following fact. Consider two points $X$ and $Y$ on Minkowski space, with $-t^{2}=X \cdot X$ and $-u^{2}=Y \cdot Y$. Of crucial importance is the distance $d$ between $X$ and $Y$, which is given by

$$
d^{2}=(X-Y) \cdot(X-Y) \text {. }
$$

For simplicity we assume that $t^{2}$ and $u^{2}$ have the same sign. Then $\tilde{X}=X /|t|$ and $\tilde{Y}=Y /|u|$ are coordinates on the same (anti)de Sitter space. Clearly,

$$
d^{2}=-u^{2}-t^{2}-2|u t| \tilde{X} \cdot \tilde{Y}
$$

Now the geodesic distance on (anti)de Sitter space is a function of $\tilde{X} \cdot \tilde{Y}$ only, and we see that the geodesic distance in Minkowski space is a function of $t, u$ and the geodesic distance in (anti)de Sitter space only. This fact is essential in relating Minkowski space quantities to correlation functions in the CFT's.

The emerging picture is rather intriguing. It suggests that it must be possible to completely re-formulate the Quantum Field Theory in Minkowski space entirely in terms of correlation functions of a set of conformal operators associated with the past and future of the light-cone. We consider this as a step towards a better understanding of the holography (well established in anti-de Sitter spacetime) in asymptotically flat spacetimes.

An important element in formulation of a holographic duality is the identification of the underlying conformal symmetry. In asymptotically anti-de Sitter spaces this symmetry arises as asymptotic symmetry near the time-like infinity [10]. The class of asymptotically flat spaces and the respective symmetries near the null-infinity (where the holographic dual could naively be thought to live) were studied long ago in [11] (see also [12] and [13] for a related discussion of the space-like infinity). The group of symmetries is the so-called BMS group, an infinite dimensional abelian group, which does not contain the relevant conformal group. The idea pushed forward in this paper is to look at the infinity of the light-cone and uncover the asymptotic symmetries there. The allowed set of spaces is larger than the set of asymptotically flat spaces, and the group of asymptotic symmetries contains the BMS group but also the conformal group. The appearance of the conformal symmetry in this case is quite transparent. It is just an extension of the well-studied conformal symmetry near the boundary of a anti-de Sitter slice to a symmetry near the boundary of the light-cone in Minkowski space. The price to pay for all this is that translation invariance of Minkowski space is not manifest, but takes a much more complicated form.

The outline of this paper is as follows. In section 2 we discuss in more detail the slicing of Minkowski space-time and its properties. In section 3 we consider free massless scalar fields, and their reduction on each of the slices. In section 4 we consider the finite part of the on-shell action for a free scalar field, and will see that the structure suggests the 
presence of conformal correlation functions in Minkowski space quantities. In section 5 we will make the relation between correlation functions in the conformal field theory and bulk quantities more precise, and we use this to reconstruct the Minkowski space S-matrix and Green's function. In section 6 we look at more general spaces. We introduce an appropriate notion of asymptotically Minkowski space, analyze the symmetries that preserve these asymptotics. We also examine the way conformal symmetries are implemented and whether it is meaningful to view the infinite set of conformal operators as fields living in a space with one additional dimension, and comment on the meaning of translation invariance and the BMS group. In section 7 we study gravity in Minkowski space, and how the space-time metric is encoded in data on the two spheres. Finally, we present some conclusions and further speculations.

\section{Slicing Minkowski space}

In this section we consider the foliation of Minkowski spacetime with (positive or negative) constant curvature subspaces that is naturally associated to the light-cone structure of the space-time. Let us consider $(d+2)$-dimensional Minkowski space with coordinates $X_{0}, X_{1}, \ldots, X_{d+1}$ and metric

$$
d s^{2}=-d X_{0}^{2}+d X_{1}^{2}+\ldots+d X_{d+1}^{2} .
$$

The light-cone defined as

$$
\mathcal{C}: \quad-X_{0}^{2}+X_{1}^{2}+\ldots+X_{d+1}^{2}=0
$$

naturally splits the space-time in three regions

1. $\mathcal{D}: \quad-X_{0}^{2}+X_{1}^{2}+\ldots+X_{d+1}^{2}>0$, the region lying outside the light-cone $\mathcal{C}$;

2. $\mathcal{A}_{-}: \quad-X_{0}^{2}+X_{1}^{2}+\ldots+X_{d+1}^{2}<0, \quad X_{0}<0$, the region lying inside the past light-cone $\mathcal{C}_{-}\left(X_{0}<0\right)$

3. $\mathcal{A}_{+}: \quad-X_{0}^{2}+X_{1}^{2}+\ldots+X_{d+1}^{2}<0, \quad X_{0}>0$, the region lying inside the future light-cone $\mathcal{C}_{+}\left(X_{0}>0\right)$.

In each region Minkowski space can be foliated with surfaces of constant curvature. In region $\mathcal{A}_{-}$( or $\left.\mathcal{A}_{+}\right)$these are the surfaces described by the equation

$$
-X_{0}^{2}+X_{1}^{2}+\ldots+X_{d+1}^{2}=-t^{2}
$$

for a constant $t$. The values of the time coordinate $t$ can be arranged in such a way that region $\mathcal{A}_{-}$is covered by $-\infty<t<0$ and region $\mathcal{A}_{+}$by $0<t<+\infty$. As is well known, each of these surfaces with the metric induced from (2.1) is Euclidean anti-de Sitter space, which is a maximally symmetric hyperbolic space. The metric on $\mathcal{A}$ foliated by the surfaces (2.2) is

$$
\begin{aligned}
& d s^{2}=-d t^{2}+t^{2} d s_{H_{d+1}}^{2} \\
& d s_{H_{d+1}}^{2}=d y^{2}+\sinh ^{2} y d \omega_{d}^{2}
\end{aligned}
$$

where $d s_{H_{d+1}}^{2}$ is the standard metric on Euclidean anti-de Sitter space; we adopt coordinates $(y, \theta)$ on $H_{d+1}$ such that $y$ is the radial coordinate and $\{\theta\}$ are the angle coordinates on the unit d-sphere with metric $d \omega_{d}^{2}$. 
Similarly, outside the light-cone, region $\mathcal{D}$ can be sliced with with de Sitter hypersurfaces,

$$
-X_{0}^{2}+X_{1}^{2}+\ldots+X_{d+1}^{2}=r^{2},
$$

which for any constant $r$ is a maximally symmetric Lorentzian space with negative constant curvature. Using $r$ as a new "radial" coordinate we find that the Minkowski metric on this foliation takes the form

$$
\begin{aligned}
& d s^{2}=d r^{2}+r^{2} d s_{d S_{d+1}}^{2} \\
& d s_{d S_{d+1}}^{2}=-d \tau^{2}+\cosh ^{2} \tau d \omega_{d}^{2}
\end{aligned}
$$

where $d s_{d S_{d+1}}^{2}$ is the metric on $(d+1)$-dimensional Lorentzian de Sitter space covered by the global coordinates $(\tau, \theta)$, with $\tau$ playing the role of time and with $\{\theta\}$ coordinates on the unit d-sphere.

Each of the metrics (2.3) and (2.5) covers only a part of Minkowski space. In order to cover the whole spacetime we need metrics on three regions: $\mathcal{A}_{-}, \mathcal{A}_{+}$and $\mathcal{D}^{\ddagger}$. It is also possible to introduce a closely related global coordinate system $\left(x_{+}, x_{-}, \theta\right)$ which covers all of spacetime and in terms of which the metric takes the well-known form [14]

$$
d s^{2}=-d x_{+} d x_{-}+\frac{1}{4}\left(x_{+}-x_{-}\right)^{2} d \omega_{d}^{2} .
$$

In region $\mathcal{A}$ the relation between the coordinates $(t, y, \theta)$ and the global coordinates is given by

$$
x_{+} x_{-}=t^{2}, \frac{x_{-}}{x_{+}}=e^{2 y} .
$$

In region $\mathcal{A}_{-}$both $x_{+}$and $x_{-}$are negative while in region $\mathcal{A}_{+}$they are both positive. In region $\mathcal{D}$ the relation between the coordinates $(r, z, \theta)$ and the coordinates $\left(x_{+}, x_{-}, \theta\right)$ reads

$$
x_{+} x_{-}=-r^{2}, \frac{x_{+}}{x_{-}}=-e^{2 \tau} .
$$

One can see that in this region $x_{+} x_{-}<0$. The light-cone $\mathcal{C}$ is defined by equation $x_{+} x_{-}=0$ so that its component $\mathcal{C}_{-}$corresponds to $x_{+}=0$ while the equation for the future light-cone $\mathcal{C}_{+}$is $x_{-}=0$.

It is interesting to determine the boundaries associated to the slicing. First of all, the past light cone $\mathcal{C}_{-}$has a $d$-dimensional sphere $S_{d}^{-}$as a boundary (lying at negative infinite value of the time coordinate $X_{0}$ ). In terms of new projective coordinates $\xi_{1}=X_{1} / X_{0}, \ldots$, $\xi_{d+1}=X_{d+1} / X_{0}$ the equation of the sphere is

$$
\xi_{1}^{2}+\ldots+\xi_{d+1}^{2}-1=0 .
$$

Similarly, the boundary of the future cone $\mathcal{C}_{+}$is a sphere $S_{d}^{+}$. The hyperbolic surface (2.2), as is well known and much used in the context of the AdS/CFT correspondence,

\footnotetext{
${ }^{\ddagger}$ Strictly speaking, we also need to take the lightcone itself into account. However, this is a subspace of measure zero, and we will therefore ignore it, but it is indirectly present in the form of the boundary conditions we will choose
} 


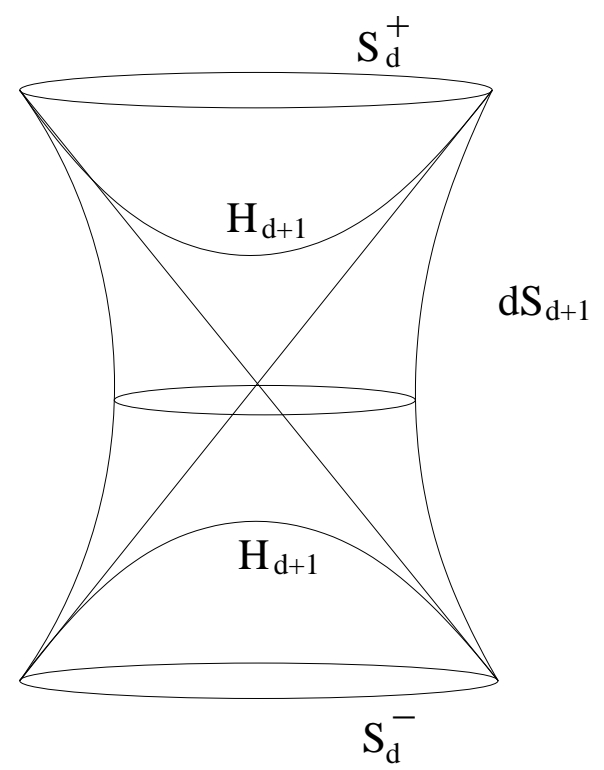

Fig. 1: Inside light-cone $(\mathrm{d}+2)$-dimensional Minkowski space-time can be sliced with Euclidean anti-de Sitter spaces $H_{d+1}$ while out-side the light-cone the appropriate slices are Loretzian de Sitter spaces $d S_{d+1}$. The only boundaries of the slices are either sphere $S_{d}^{-}$or sphere $S_{d}^{+}$lying at infinity of the light-cone.

has a d-dimensional sphere as a boundary. It is now important to note that it is again the same sphere $S_{d}^{-}$. Indeed, in terms of the coordinates $\left\{\xi_{k}\right\}$ equation (2.2) becomes

$$
\xi_{1}^{2}+\ldots+\xi_{d+1}^{2}-1=-t^{2} / X_{0}^{2}
$$

which (for any constant $t$ ) approaches the above written equation for $S_{d}^{-}$in the limit where $X_{0} \rightarrow-\infty$. Similarly, we can see that the boundary of a slice (2.2) in region $\mathcal{A}_{+}$is the sphere $S_{d}^{+}$, the boundary of the future cone $\mathcal{C}_{+}$. de Sitter space defined by equation (2.4) has two boundaries: a sphere lying in the infinite past and a sphere lying in the infinite future. It is clear that for any slice (2.4) the boundaries are again respectively the spheres $S_{d}^{-}$and $S_{d}^{+}$. Thus the d-spheres $S_{d}^{-}$and $S_{d}^{+}$are the only boundaries that appear in the slicing that we are considering, and they therefore play an important role in the remainder of the paper. If we look at the initial value problem, we observe that the hypersurfaces (2.2) are not Cauchy surfaces. Initial data on such a surface is not sufficient to determine the time-evolution everywhere in Minkowski space. A complete set of initial data should necessarily also include data on a de Sitter hypersurface (2.4). In this paper we shall however argue that all sufficient information can be actually given on a lower dimensional subspace:

All information on (d+2)-dimensional Minkowski space-time is holographically described by some data on the two d-spheres $S_{d}^{-}$and $S_{d}^{+}$.

In the following sections we shall give arguments in support of this statement. Here we just note that this may not seem to be too surprising. Indeed, we already know that all information needed for anti-de Sitter space is contained in certain data on its boundary. The same is true for de Sitter space (at least classically), only in this case the boundary has two components, one at past and one at future infinity. In the foliation of Minkowski 
space just described all (anti)-de Sitter hypersurfaces have the same boundaries, $S_{d}^{-}$and $S_{d}^{+}$, and it is natural to expect that this is where all needed information is stored.

Ending this section we note that the isometry group $\mathrm{O}(\mathrm{d}+1,1)$ of Minkowski spacetime acts on the spheres $S_{d}^{-}$(or $S_{d}^{+}$) as a group of conformal symmetries. In section 6 we find explicitly the form of the bulk diffeomorphisms generating conformal transformations on the boundary of the light-cone. The conformal structure associated with the boundary of (anti-)de Sitter space then appears to be a particular manifestation of the conformal structure associated with the infinity of the light-cone. The relation between a Minkowski metric with Lorentzian signature, the metric on Euclidean AdS and the conformal structure on the sphere was earlier studied in [15], a popular reference in the literature on the AdS/CFT correspondence. The relevance of Minkowski space has however not received much attention in subsequent studies.

\section{Scalar Field in Minkowski space-time}

Consider a scalar field with equation of motion

$$
\left(\nabla^{2}-M^{2}\right) \phi=0
$$

on $(\mathrm{d}+2)$-dimensional Minkowski spacetime and use the slicing with constant curvature spaces described in the previous section to see whether the field can be equivalently described in terms of data living on the slice. First we analyze the massless case and take the region $\mathcal{A}_{-}$with metric (2.3), $t$ being the time-like coordinate taking negative values. The analysis for the region $\mathcal{A}_{+}$is similar. In terms of the metric (2.3) the field equation (3.1) reads

$$
\Delta_{H_{d+1}} \phi-(d+1) t \partial_{t} \phi-t^{2} \partial_{t}^{2} \phi=0
$$

We can separate variables in this equation by taking $\phi$ of the form $\phi \sim(-t)^{-\lambda(\mu)} \phi_{\mu}(y, \theta)$ where $(y, \theta)$ is the set of coordinates on the Euclidean hyperbolic space $H_{d+1}$ (which is the same as Euclidean anti-de Sitter space). For a given $\mu$ the functions $\phi_{\mu}(x)$ satisfy the massive field equation on $H_{d+1}$

$$
\left(\Delta_{H_{d+1}}-\mu^{2}\right) \phi_{\mu}(x)=0
$$

and the parameter $\mu$ thus plays the role of mass. The original $(d+2)$-dimensional field $\phi(t, x)$ reduces to a set of massive fields on $(d+1)$-dimensional Euclidean anti-de Sitter space. The relation between $\mu$ and the parameter $\lambda(\mu)$ can be read from (3.2) and is given $\mathrm{by}^{\S}$

$$
\lambda_{ \pm}=\frac{d}{2} \pm \sqrt{\mu^{2}+\frac{d^{2}}{4}} .
$$

Thus, there are exactly two independent solutions, $(-t)^{-\lambda_{+}} \phi^{+}(x)+(-t)^{-\lambda_{-}} \phi^{-}(x)$. When $\mu^{2}+\frac{d^{2}}{4}=0$ the independent solutions are $t^{-\frac{d}{2}}$ and $t^{-\frac{d}{2}} \ln t$. Normally, we would choose one of the solutions by imposing appropriate boundary conditions. One possible condition is

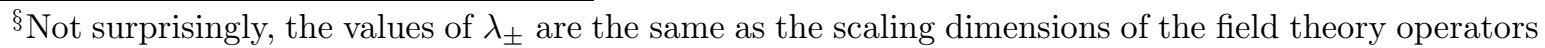
corresponding to $\phi_{\mu}$ in the AdS/CFT correspondence.
} 
regularity at $t=0$ (i.e. near the past light-cone $\mathcal{C}_{-}$) which selects only negative values of $\lambda$. Note that at infinity (when $t$ goes to $-\infty$ ) we pick up the most divergent solution. This is similar to the usual story in anti-de Sitter space. Another possible condition is the regularity of the action in a neighbourhood of $t=0$,

$$
W[\phi]=\int_{t \rightarrow 0} t^{d+1} \phi(x, t) \partial_{t} \phi(x, t)
$$

This gives the condition $\operatorname{Re} \lambda<\frac{d}{2}$. In case $\operatorname{Re} \lambda=\frac{d}{2}$ the expression (3.5) does not contain growing (as $t$ goes to zero) terms but there are oscillating terms. These terms are allowed in quantum field theory since they correspond to propagating waves, and they will also play an important role in the remainder of this paper.

Note that the real part of $\lambda_{+}$is always positive and thus can not provide us with a regular solution at $t=0$. For $\lambda_{-}$, depending on the value of $\mu^{2}$ in (3.3), we have three different cases:

1. $\mu^{2}>0$, then $\lambda_{-}$is negative and the mode $(-t)^{-\lambda_{-}}$is regular at $t=0$.

2. $-\frac{d^{2}}{4}<\mu^{2}<0, \lambda_{-}$is positive while still real. There are no solutions regular at $t=0$ in this case. However, $\lambda_{-}<\frac{d}{2}$ and hence the mode $(-t)^{-\lambda_{-}}$leads to a regular action (at $t=0)$.

3. $\mu^{2}<-\frac{d^{2}}{4}$, i.e. $\mu^{2}=-\frac{d^{2}}{4}-\alpha^{2}$, then both $\lambda_{+}$and $\lambda_{-}$are complex: $\lambda_{ \pm}=\frac{d}{2} \pm i \alpha$. The action has only oscillating terms at $t=0$ in this case and both $(-t)^{-\lambda_{+}}$and $(-t)^{-\lambda_{-}}$ are allowed.

According to the AdS/CFT correspondence, to each field on anti-de Sitter (with mass $\mu$ ) satisfying equation (3.3) and the Breitenlohner-Freedman bound $\mu^{2}>-d^{2} / 4$ there exists a dual conformal operator living on the boundary of anti-de Sitter space (i.e. on the sphere $S_{d}^{-}$). The conformal dimension of the operator is the largest root of the equation $h(h-d)=\mu^{2}$, for positive $\mu^{2}$ it is

$$
h_{+}=\frac{d}{2}+\sqrt{\frac{d^{2}}{4}+\mu^{2}} .
$$

Unitarity requires the conformal weight to be real and that in turn implies that $\mu^{2}$ has to satisfy the Breitenlohner-Freedman bound. We thus see that this bound also has a clear meaning from the higher dimensional Minkowski space prospective.

Notice that the propagating modes correspond to case 3 and are thus described by complex conformal weights, $h_{ \pm}=\frac{d}{2} \pm i \alpha$, with $\alpha$ an arbitrary real parameter. The standard plane wave is described in terms of the metric (2.1) as $e^{i k_{\mu} X^{\mu}}$, where $k^{2}=0$. In the coordinates $\{t, y, \theta\}$ it takes the form $e^{i p(y, \theta) t}$, where $p(y, \theta)$ is some function of the coordinates $y$ and $\theta$. These modes can be decomposed in the basis formed by functions $t^{-\lambda}$ where $\lambda$ is complex. This is seen from the inverse Mellin type transform [16]

$$
e^{i p t}=\frac{1}{2 \pi i} \int_{c-i \infty}^{c+i \infty} d \lambda t^{-\lambda} \frac{i^{\lambda} \Gamma(\lambda)}{(p+i \epsilon)^{\lambda}}
$$

where $\Gamma(\lambda)$ is the Gamma function. Thus, to describe plane waves, or more generally any solution of the free field equations which is normalizable in the sense of plane waves we only need the modes with $\lambda=\frac{d}{2} \pm i \alpha$. Normally, such values of the conformal weights violate unitarity in a dual CFT. However, it is important to keep in mind that we are here discussing Euclidean AdS as a building block of a Minkowski-signature theory, and we do 
not intend to analytically continue the results to Lorentzian AdS. Therefore, it is not clear whether the notion of unitarity that is normally inherited from Lorentzian AdS has any relation to the notion of unitarity in Minkowski space. Since Minkowski space physics carries a unitary representation of the Lorentz group $S O(d+1,1)$, we would actually expect that this is the group that is unitarily represented, and for this group complex weights are not problematic, they correspond to the principal series representation. All this is very similar to a corresponding discussion for de Sitter space given in [17. We therefore view the results in this paper as providing further motivation for the claim in [17. that the appropriate notion of unitarity in de Sitter space is one where $S O(d+1,1)$ is unitarily implemented, and complex weights therefore do not violate unitarity.

In the region $\mathcal{D}$ the story is essentially similar with the coordinate $t$ replaced by the space-like coordinate $r$. Solving equation (3.1) for the metric (2.4) we decompose solutions in terms of modes $r^{-\lambda} \phi_{m}(x)$, where $\phi_{m}(x)$ is now a solution to the field equation with mass $m$ on de Sitter space

$$
\left(\Delta_{d S_{d+1}}-m^{2}\right) \phi_{m}=0
$$

The relation between $m$ and $\lambda$ is given by

$$
\lambda_{ \pm}=\frac{d}{2} \pm \sqrt{\frac{d^{2}}{4}-m^{2}} .
$$

The analysis of modes, regularity conditions and conformal weights are similar to the analysis in $\mathcal{A}_{-}$. To a large extent the results can be obtained by analytical continuation of the anti-de Sitter mass $m^{2}$ to the de Sitter mass $\mu^{2}=-m^{2}$. In particular, the modes $r^{-\lambda_{-}}$regular on the light cone $(r=0)$ are the ones with $m^{2}<0$. In all versions of the $\mathrm{dS} / \mathrm{CFT}$ correspondence that have appeared in the literature the conformal weight of the corresponding operator on the boundary of de Sitter is

$$
h=\frac{d}{2}+\sqrt{\frac{d^{2}}{4}-m^{2}} .
$$

The modes with $0<m^{2}<\frac{d^{2}}{4}$ are not regular at the light-cone but have finite action there. Finally, modes with $m^{2}=\frac{d^{2}}{4}+\alpha^{2}$ are oscillating at the light-cone. Both values of the conformal weight, $h_{+}=\frac{d}{2}+i \alpha$ and $h_{-}=\frac{d}{2}-i \alpha$, are allowed in this case. The latter ones are the modes that are needed to describe plane-wave normalizable solutions of the field equations.

The modes described are analytic across the light-cone so that modes with $m^{2}=\mu^{2}$ in region $\mathcal{A}$ become modes with $m^{2}=-\mu^{2}$ in region $\mathcal{D}$. In particular, this means that the limiting values $\left\{\phi_{\mu}\left[S^{-}\right]\right\} \quad\left(\left\{\phi_{\mu}\left[S^{+}\right]\right\}\right)$of the wave function at $S_{d}^{-}\left(S_{d}^{+}\right)$are the same regardless of whether the light-cone is approached from region $\mathcal{D}$ or from the region $\mathcal{A}_{-}$ $\left(\mathcal{A}_{+}\right)$. To see this explicitly note that in region $\mathcal{A}_{-}$close to the boundary at infinity of the past light-cone the modes behave as $(-t)^{-\lambda_{-}} e^{-\lambda_{-} y}=\left(-x_{-}\right)^{-\lambda_{-}}$, where $x_{-}$(see (2.7)) is a coordinate in the coordinate system (2.6) covering all of spacetime. It approaches $-\infty$ as we approach the boundary at infinity of the past light-cone. On the other hand, in the region $\mathcal{D}$ the modes (with $m^{2}=-\mu^{2}$, i.e. with the same $\lambda_{-}$) behave in the same region as $r^{-\lambda_{-}} e^{\lambda \tau}=\left(-x_{-}\right)^{-\lambda_{-}}$, where the equality follows from (2.8). The past light-cone is the surface where $x_{+}=0$. We see that the modes are analytic across this surface and 
describe a wave configuration propagating along the past light-cone. In a similar fashion one finds that the modes are also analytic across the future light-cone.

Let us now turn on the mass in equation (3.1). The decomposition of the bulk field over a set of massive fields living on the slice is valid also in this case. However the radial functions in the decomposition (we take region $\mathcal{D}$ for concreteness)

$$
\phi(r, x)=\int d m \frac{1}{r^{d / 2}} K_{\nu(m)}(M r) \phi_{m}(x)
$$

are now expressed in terms of the MacDonald function $K_{\nu}(M r)$, where $\nu=\sqrt{\frac{d^{2}}{4}-m^{2}}$. The integration over $m$ in (3.11) should be understood as symbolic, the range of values and measure on that space may depend on the class of functions we want to consider. Each massive field on the slice has a holographic description in terms of conformal operators on the boundary of the slice. The relation between the conformal weight and the mass $m$ is given by (3.10). In this sense there is no difference with the massless bulk field considered above. A plane wave solution of the massive field equation in the bulk can also be decomposed in terms of the radial functions (3.11). The relevant integral transform is known in the mathematical literature as the Kantorovich-Lebedev transform. The analog of equation (3.7) is

$$
e^{i p r}=\int_{0}^{+\infty} d \alpha r^{-d / 2} K_{i \alpha}(M r) f(p, \alpha)
$$

where the function $f(p, \alpha)$ is expressed in terms of a hypergeometric function as follows

$$
f(p, \alpha)=\frac{2 \alpha}{\pi^{\frac{3}{2}}} \sinh \alpha \frac{1}{(2 M)^{\frac{d}{2}}} \frac{\Gamma\left(\frac{d}{2}+i \alpha\right) \Gamma\left(\frac{d}{2}-i \alpha\right)}{\Gamma\left(\frac{d+1}{2}\right)} F\left(\frac{d}{2}+i \alpha, \frac{d}{2}-i \alpha, \frac{d+1}{2},\left(\frac{1}{2}+\frac{i p}{2 M}\right)\right)
$$

Interestingly, it can be brought to a compact form

$$
f(p, \alpha)=\frac{2(2 \pi)^{d / 2}}{\Gamma(i \alpha) \Gamma(-i \alpha)} M^{-d / 2} D_{\frac{d}{2}+i \alpha}\left(i \frac{p}{M}\right)
$$

in terms of a de Sitter invariant Green's function $D_{\lambda}(z)$ to be defined in section 5 ( $z$ in that case would be related to the geodesic distance on a de Sitter slice). The appearance of this function in the Kantorovich-Lebedev transform (3.12) is rather mysterious. Since massive fields in the bulk do not lead to any immediate new conceptual issues but are mainly technically more complicated, we will restrict our attention to massless fields in the remainder of this paper.

Summarizing our progress so far, we have found that the scalar (massive or massless) field propagating in Minkowski space is equivalently described in terms of an infinite set of massive fields living (depending on the region of Minkowski space) on anti-de Sitter or de Sitter space. The original scalar field can thus be decomposed in terms of massive fields on (anti-) de Sitter. This is a sort of spectral decomposition since it involves an integration over the mass $\mu$ (or $m$ ), which therefore plays the role of the spectral parameter. The explicit form of this decomposition will be studied in section 5 .

\footnotetext{
`Another possible Bessel function (Infeld function $I_{\nu}(M r)$ ) is exponentially growing at infinity and thus omitted.
} 
Several of the equations and decompositions given in this and later sections have also been obtained in the papers [18, 19], but we are not aware of any attempt to use de Sitter and AdS slices to try to find a holographic dual description of Minkowski space. Similarly, one can consider massless higher spin fields, but the generalization is rather straightforward and will not be discussed in this paper.

As we know from the AdS/CFT correspondence any massive field on Euclidean anti-de Sitter space is completely described by fixing Dirichlet data on the boundary of the space, which in the present case is the sphere $S_{d}^{-}$(or $S_{d}^{+}$for the region $\mathcal{A}_{+}$). One has to be a bit careful in applying the standard AdS/CFT formalism, because in our case we have complex conformal weights and there is no longer a separation of fields in normalizable and non-normalizable modes. The analog statement for de Sitter space is that the initial-time data can be fixed on either the past or future infinity surface which is again respectively $S_{d}^{-}$ or $S_{d}^{+}$. This suggests that the Cauchy problem in Minkowski space becomes the problem of reconstructing the values of a field everywhere in terms of known data $\left\{\phi_{\mu}\left[S^{-}\right]\right\}$given on the past infinity $\left(S_{d}^{-}\right)$of the light cone. The explicit form of this reconstruction will be given in subsection 5.2. Having in mind the Quantum Mechanical picture we would expect that the quantum in-state should be associated with data on $S_{d}^{-}$while the outstate should be defined in terms of the data $\left\{\phi_{\mu}\left[S^{+}\right]\right\}$on the future infinity $\left(S_{d}^{+}\right)$of the light cone. In the next sections we will elaborate more on this picture.

\section{Light-cone conformal correlation functions}

In this section we show that in a manner similar to the AdS/CFT correspondence [5] there naturally appear conformal field theory correlation functions on the boundary of the light-cone in Minkowski spacetime. Actually, the discussion in this section should be taken with a grain of salt and be viewed as providing some inspiration for the results in the remainder of the paper.

Consider the action of the scalar field on $\mathcal{A}_{-}$. If the field is a solution of the equations of motion the action reduces to the boundary term

$$
W=\int_{t \rightarrow-\infty}(-t)^{d+1} \sqrt{\gamma} \phi(x, t) \partial_{t} \phi(x, t)
$$

where $x \equiv(y, \theta)$ are coordinates on the Anti-de Sitter slice, $H_{d+1}$. We decompose fields in terms of modes as we did in the previous section,

$$
\phi(x, \tau)=\sum_{\left(h_{+}, h_{-}\right)}(-t)^{-h_{+}} \phi^{(+)}(x)+(-t)^{-h_{-}} \phi^{(-)}(x)
$$

where we sum (or, rather, take an integral if $h_{ \pm}$are continuous) over all possible pairs $\left(h_{+}, h_{-}\right)$. In terms of the massive fields (with mass $\mu$ ) on anti-de Sitter space $H_{d+1}, h_{ \pm}$ are the roots of the equation $h(h-d)=\mu^{2}\left(h_{+}+h_{-}=d, \mu^{2}=-h_{+} h_{-}\right)$. In this section we do not specify the sign of $\mu^{2}$. In the decomposition (4.2) a priori all possible (both real and complex) conformal weights should be taken into account, but, as we discussed in the previous section, plane wave normalizability restricts the conformal weights to $h_{ \pm}=\frac{d}{2} \pm i \alpha$. Next, we substitute (4.2) into (4.1) and take the limit $t \rightarrow-\infty$. In general the resulting expression has infinite, oscillating and finite terms

$$
W=\sum_{h+h^{\prime}=d} \int_{H_{d+1}} h^{\prime} \phi_{h}(x) \phi_{h^{\prime}}(x)+\sum_{\operatorname{Re}\left(d-h-h^{\prime}\right)>0} h^{\prime}(-t)^{\left(d-h-h^{\prime}\right)} \int_{H_{d+1}} \phi_{h}(x) \phi_{h^{\prime}}(x)
$$


where the infinite and oscillating terms are collected in the second sum. We are interested in the finite one,

$$
W_{f i n}=\sum_{\left(h_{+}, h_{-}\right)} d \int_{H_{d+1}} \phi^{(+)}(x) \phi^{(-)}(x)
$$

where the summation (integration) runs over all possible pairs $\left(h_{+}, h_{-}\right)$.

Before proceeding, we point out two problems with the analysis in this section. First, we have to drop some infinite and oscillating terms in order to get (4.3). In the spirit of the usual AdS/CFT correspondence, such a procedure would be viewed as the holographic version of renormalization, but here such an interpretation is problematic, because the subleading terms in the $t$ expansion are non-local functionals of the leading term, and therefore the infinities are non-local in nature [20]. However, what we really want to do is holography in each of the AdS slices and keep the $t$-dependence exactly, and then this issue will not arise. Besides this, there are problems evaluating on-shell actions for scalar fields on de Sitter slices, and probably also for fields on Euclidean anti-de Sitter space with complex conformal weights, as discussed in [17. Both problems will not arise in the next section where we use an S-matrix type approach to the Minkowski/CFT duality, and as we said above, this section should mainly be viewed as providing some inspiration.

Continuing with the analysis, we recall that each field $\phi^{(+)}(x)$ and $\phi^{(-)}(x)$ is a solution of the scalar field equation

$$
\left(\Delta-\mu^{2}\right) \phi^{( \pm)}(x)=0
$$

with mass $\mu^{2}=-h_{+} h_{-}$on $H_{d+1}$. The coordinates $x=(\theta, y)$ are such that $y=\infty$ corresponds to the boundary of $H_{d+1}$ and $(\theta)$ are the angle coordinates on the asymptotic sphere $S_{d}^{-}$.

Near the boundary of $H_{d+1}$ there exists a decomposition

$$
\begin{aligned}
& \phi^{(+)}(x)=e^{-h_{-} y} \phi_{-}^{(+)}(\theta)+e^{-h_{+} y} \phi_{+}^{(+)}(\theta) \\
& \phi^{(-)}(x)=e^{-h_{-} y} \phi_{-}^{(-)}(\theta)+e^{-h_{+} y} \phi_{+}^{(-)}(\theta)
\end{aligned}
$$

familiar from the AdS/CFT duality [5]. If $h_{+}$and $h_{-}$are real the term $e^{-h_{-} y}$ dominates and the functions $\phi_{-}^{(+)}(\theta), \phi_{-}^{(-)}(\theta)$ are the usual choices for the Dirichlet boundary condition at the boundary of $\mathrm{AdS}$ space $H_{d+1}$. If the conformal weights are complex any function $\phi_{-}^{( \pm)}(\theta)$ or $\phi_{+}^{( \pm)}(\theta)$ can be used as a boundary condition. The solutions of the massive scalar field equation on $H_{d+1}$ can then chosen to be

$$
\begin{aligned}
& \phi^{(+)}(\theta, y)=\int_{S_{d}} d \mu\left(\theta^{\prime}\right) G_{-}\left(y, \theta, \theta^{\prime}\right) \phi_{+}^{(+)}\left(\theta^{\prime}\right), \\
& \phi^{(-)}(\theta, y)=\int_{S_{d}} d \mu\left(\theta^{\prime}\right) G_{+}\left(y, \theta, \theta^{\prime}\right) \phi_{-}^{(-)}\left(\theta^{\prime}\right),
\end{aligned}
$$

where $d \mu(\theta)$ is the standard measure on a unit radius d-sphere, and we will from now on use the notation $\gamma\left(\theta, \theta^{\prime}\right)$ for the geodesic distance on a d-sphere between points with coordinates $\theta$ and $\theta^{\prime}$. We will ignore the fact that for complex weights the fields in (4.4) are generically complex, and one should really add the complex conjugate fields in the right hand side. Again, the results here should be taken with a grain of salt. The kernels

$$
\begin{aligned}
& G_{h}\left(y, \theta, \theta^{\prime}\right)=\frac{g(h)}{\left(\cosh y-\sinh y \cos \gamma\left(\theta, \theta^{\prime}\right)\right)^{h}}, \\
& g(h)=(4 \pi)^{-\frac{d}{2}} \frac{\Gamma(h)}{\Gamma\left(h-\frac{d}{2}\right)},
\end{aligned}
$$


can be interpreted as bulk-boundary propagators on an anti-de Sitter slice. For large $y$ they behave as follows (see Appendix A for details)

$$
\begin{aligned}
& \lim _{y \rightarrow \infty} G_{+}\left(y, \theta, \theta^{\prime}\right)=\left(e^{-h_{-} y} \delta^{(d)}\left(\theta, \theta^{\prime}\right)+e^{-h_{+} y} K_{+}\left(\theta, \theta^{\prime}\right)\right)\left(1+\mathcal{O}\left(e^{-2 y}\right)\right) \\
& \lim _{y \rightarrow \infty} G_{-}\left(y, \theta, \theta^{\prime}\right)=\left(e^{-h_{+} y} \delta^{(d)}\left(\theta, \theta^{\prime}\right)+e^{-h_{-} y} K_{-}\left(\theta, \theta^{\prime}\right)\right)\left(1+\mathcal{O}\left(e^{-2 y}\right)\right),
\end{aligned}
$$

where $\delta^{(d)}\left(\theta, \theta^{\prime}\right)$ is the delta-function on the d-sphere. The kernels $K_{-}\left(\theta, \theta^{\prime}\right)$ and $K_{+}\left(\theta, \theta^{\prime}\right)$ are defined as

$$
K_{ \pm}\left(\theta, \theta^{\prime}\right)=\frac{2^{h_{ \pm}} g\left(h_{ \pm}\right)}{\left(1-\cos \gamma\left(\theta, \theta^{\prime}\right)\right)^{h_{ \pm}}}
$$

and are inverse to each other

$$
\int d \mu(\theta) K_{-}\left(\theta, \theta^{\prime}\right) K_{+}\left(\theta, \theta^{\prime \prime}\right)=\delta^{(d)}\left(\theta^{\prime}, \theta^{\prime \prime}\right)
$$

Thus, from (4.4) we have that

$$
\begin{aligned}
& \phi_{-}^{( \pm)}(\theta)=\int d \mu\left(\theta^{\prime}\right) K_{-}\left(\theta, \theta^{\prime}\right) \phi_{+}^{( \pm)}\left(\theta^{\prime}\right) \\
& \phi_{+}^{( \pm)}(\theta)=\int d \mu\left(\theta^{\prime}\right) K_{+}\left(\theta, \theta^{\prime}\right) \phi_{-}^{( \pm)}\left(\theta^{\prime}\right) .
\end{aligned}
$$

The idea is to substitute (4.2)-(4.4) into the action (4.1), first take the integration over $y$ and then take the limit of infinite $t$. Note, that additionally to the divergences at large $t$ there could be extra divergences when the integration over $y$ is being taken.

Another important point is to observe that

$$
\int_{S_{d}} d \mu\left(\theta^{\prime}\right) \int_{0}^{\infty} d y \sinh ^{d}(y) G_{h}\left(y, \theta^{\prime}, \theta\right) G_{h^{\prime}}\left(y, \theta^{\prime}, \theta^{\prime \prime}\right) \sim \delta^{(d)}\left(\theta, \theta^{\prime \prime}\right) \delta\left(d-h-h^{\prime}\right)
$$

where we assumed that $h$ and $h^{\prime}$ take values on the complex line $d / 2+i \alpha$, and possibly omitted some regular terms in the right hand side. We suspect these regular terms are not there, but have not been able to prove this. The delta-function in (4.10) can be recognized in the asymptotics (4.6) of the kernels $G_{h}\left(y, \theta, \theta^{\prime}\right)$ (note, that we also omitted the terms proportional to $\delta\left(h-h^{\prime}\right)$ which contribute to the oscillating part of the action). With the help of this formula we find that the finite part of the action, after the integrations over $t$ and $y$ have been performed, is given by

$$
\begin{aligned}
& W_{f i n} \propto \sum_{\left(h_{+}+h_{-}=d\right)} \int_{S^{-}} \phi_{-}^{(-)}(\theta) \phi_{+}^{(+)}(\theta) \\
& =\sum_{\left(h_{+}+h_{-}=d\right)} \int_{S^{-}} \int_{S^{-}} d \mu(\theta) \phi_{-}^{(-)}(\theta) K_{+}\left(\theta, \theta^{\prime}\right) \phi_{-}^{(+)}\left(\theta^{\prime}\right) d \mu\left(\theta^{\prime}\right) .
\end{aligned}
$$

For complex conformal weights we have $\left[\phi_{-}^{(-)}\right]^{*}=\phi_{+}^{(+)}$and $\left[\phi_{+}^{(-)}\right]^{*}=\phi_{-}^{(+)}$so that various complex conjugates will appear in (4.11). If we were to follow the usual AdS/CFT story, we would interpret the functions $\phi_{-}^{(-)}(\theta)$ and $\phi_{-}^{(+)}(\theta)$ as sources coupled to dual conformal operators $\mathcal{O}_{+}^{(-)}(\theta)$ and $\mathcal{O}_{+}^{(+)}(\theta)$ respectively (both with conformal weight $h_{+}$). Equation (4.11) would then indicate that there is a non-trivial correlation function between the 
operators $O_{+}^{(+)}(\theta)$ and $O_{+}^{(-)}(\theta)$ and that it takes the form of a conformal two-point function on the d-sphere. Again, this is all suggestive but quite imprecise.

A similar analysis can be done in regions $\mathcal{A}_{+}$and $\mathcal{D}$. By finally considering the action on all of Minkowski space we arrive at a functional $W_{f i n}\left[\phi_{h}\left[S^{-}\right], \phi_{h}\left[S^{+}\right]\right]$of data $\phi_{h}\left[S^{-}\right]$ and $\phi_{h}\left[S^{+}\right]$on the two d-spheres $S^{-}$and $S^{+}$respectively. The variation with respect to $\phi_{h}\left[S^{-}\right]$and then with respect to $\phi_{h}\left[S^{+}\right]$is naturally interpreted as a correlation function between operators living on $S^{+}$and $S^{-}$. The interesting question arises whether those correlation functions have anything to do with the S-matrix in Minkowski space (once we include interactions). We will return to this and other questions in the next section, where we will set up a more precise version of the Minkowski/CFT correspondence.

\section{Green's functions and S-matrix in Minkowski spacetime}

\subsection{Propagators}

The manifestation of the conformal structure of correlation functions associated with the asymptotic boundaries of the light-cone which we studied in the previous section was suggestive. The emergence of conformal structures becomes however clearer and more precise if we study the asymptotic behavior of the propagator in Minkowski spacetime.

For simplicity we start with the free field and consider the Hadamard type propagator defined as

$$
D\left(X, X^{\prime}\right)=<0\left|\left\{\phi(X), \phi\left(X^{\prime}\right)\right\}\right| 0>\quad .
$$

In $(\mathrm{d}+2)$-dimensional Minkowski space it takes the form

$$
D\left(X, X^{\prime}\right)=\frac{\Omega_{d}}{\left(s^{2}\right)^{d / 2}} \quad, \quad s^{2}=-\left(X_{0}-X_{0}^{\prime}\right)^{2}+\left(\mathbf{X}-\mathbf{X}^{\prime}\right)^{2}
$$

where $s^{2}$ is the space-time interval between two events and $\Omega_{d}=\frac{\Gamma\left(\frac{d}{2}\right)}{4 \pi^{d / 2+1}}$.

Let both points lie in the region $\mathcal{D}$ foliated by de Sitter slices. The foliation is given by coordinates $(r, \tau, \theta)$ that are related to the coordinates of Minkowski space as follows

$$
X_{0}=r \sinh \tau, \quad \mathbf{X}=r \cosh \tau \mathbf{n}(\theta)
$$

where $\mathbf{n}(\theta)$ is d-dimensional unit vector parametrized by angle coordinates $\{\theta\}$. The distance between two points with coordinates $(r, \tau, \theta)$ and $\left(r^{\prime}, \tau^{\prime}, \theta^{\prime}\right)$ is

$$
s^{2}=r^{2}+r^{2}-2 r r^{\prime} \cos \sigma
$$

where $\sigma$ is the geodesic distance on de Sitter space between the points $(\tau, \theta)$ and $\left(\tau^{\prime}, \theta^{\prime}\right)$

$$
\cos \sigma=-\left(\sinh \tau \sinh \tau^{\prime}-\cosh \tau \cosh \tau^{\prime} \cos \gamma\left(\theta, \theta^{\prime}\right)\right)
$$

where $\gamma\left(\theta, \theta^{\prime}\right)$ is the angle between the unit vectors $\mathbf{n}(\theta)$ and $\mathbf{n}\left(\theta^{\prime}\right)$, which at the same time is the geodesic distance on the d-sphere. Note that $\sigma^{2}>0$ for space-like intervals on de Sitter space while $\sigma^{2}<0$ for time-like intervals. In the latter case $\cos \sigma$ in (5.3) and (5.4) becomes $\cosh |\sigma|$. 
Substituting (5.3) into (5.1) and expanding in powers of $r / r^{\prime}$ we recognize a generating function for Gegenbauer polynomials

$$
\left(1-2\left(r / r^{\prime}\right) \cos \sigma+\left(r / r^{\prime}\right)^{2}\right)^{-\nu}=\sum_{n=0}^{\infty} C_{n}^{\nu}(\cos \sigma)\left(r / r^{\prime}\right)^{n}
$$

The infinite sum can be represented as a single contour integral so that we have the following useful representation for the Hadamard function

$$
D\left(X, X^{\prime}\right)=-\frac{i}{2} \Omega_{d} \int_{c-i \infty}^{c+i \infty} r^{-\lambda} r^{\prime \lambda-d} C_{-\lambda}^{d / 2}(-\cos \sigma) \frac{d \lambda}{\sin (\lambda \pi)},
$$

where the constant $c$ should satisfy the condition $0<c<d$. We can choose $c=d / 2$ so that the integral is over $\lambda=d / 2+i \alpha$ where $\alpha$ changes from minus to plus infinity. These values of $\lambda$ are natural because they appear in the decomposition of plane waves as we saw in section 3 .

The propagator is now represented in terms of Gegenbauer functions which for arbitrary $\lambda$ are defined in terms of the hypergeometric function as

$$
C_{-\lambda}^{d / 2}(-z)=\frac{\Gamma(d-\lambda)}{\Gamma(1-\lambda) \Gamma(d)} F\left(d-\lambda, \lambda ; \frac{d+1}{2} ; \frac{1}{2}(1+z)\right) .
$$

When two points on a slice approach each other the geodesic distance $\sigma$ goes to zero and the argument in Gegenbauer function approaches -1. Therefore, the argument in the hypergeometric function goes to +1 . This is the point where the hypergeometric function has a singularity, $F(\alpha, \beta ; \gamma ; z) \sim(1-z)^{\gamma-\alpha-\beta}, z \rightarrow 1$. For the hypergeometric function in the definition of Gegenbauer function we have $\gamma-\alpha-\beta=-(d-1) / 2$. Thus, when $\sigma$ approaches zero Gegenbauer function in (5.5) has singularity

$$
C_{-\lambda}^{d / 2}(-\cos \sigma) \sim \frac{1}{\sigma^{d-1}}
$$

which depends only on the dimension $d$ but not on $\lambda$. This is exactly the expected behavior of Green's function. Indeed, the Gegenbauer function $C_{\lambda}^{d / 2}(-\cos \sigma)$ is related to the de Sitter invariant Green's function defined as

$$
D_{\lambda}(z)=\frac{\Gamma(\lambda) \Gamma(d-\lambda)}{(4 \pi)^{\frac{d+1}{2}} \Gamma\left(\frac{d+1}{2}\right)} F\left(d-\lambda, \lambda, \frac{d+1}{2}, \frac{1}{2}(1+z)\right),
$$

where $z=\cos \sigma$, of the field operator $\left(\Delta-\mu^{2}\right), \mu^{2}=\lambda(\lambda-d)$. The exact relation is

$$
C_{-\lambda}^{d / 2}(-z)=\frac{4 \pi^{d / 2}}{\Gamma\left(\frac{d}{2}\right)} \sin (\pi \lambda) D_{\lambda}(z)
$$

Obviously, $D_{\lambda}(z)=D_{d-\lambda}(z)$. Equation (5.5) then takes the especially simple form

$$
D\left(X, X^{\prime}\right)=\frac{1}{2 \pi i} \int_{c-i \infty}^{c+i \infty} d \lambda r^{-\lambda} r^{\prime \lambda-d} D_{\lambda}(\cos \sigma)
$$

which shows how the Green's function in $(d+2)$-dimensional Minkowski spacetime is decomposed in terms of the infinite set of Green's functions on the $(d+1)$-dimensional de Sitter slice parametrized by $\lambda$. 
In region $\mathcal{A}$, inside the light-cone, we choose the coordinate system $(t, y, \theta)$ as follows

$$
X_{0}=t \cosh y \quad, \quad \mathbf{X}=t \sinh y \mathbf{n}(\theta)
$$

The Minkowski distance in this region takes the form

$$
s^{2}=-\left(t^{2}+t^{\prime 2}-2 t t^{\prime} \cosh \sigma\right),
$$

where $\sigma$ is the geodesic distance on Euclidean anti-de Sitter space defined as

$$
\cosh \sigma=\cosh y \cosh y^{\prime}-\sinh y \sinh y^{\prime} \cos \gamma\left(\theta, \theta^{\prime}\right)
$$

and as before $\gamma\left(\theta, \theta^{\prime}\right)$ is the geodesic distance on the unit d-sphere. The propagator in this region has a decomposition similar to (5.7),

$$
D\left(X, X^{\prime}\right)=\frac{(-i)^{d}}{2 \pi i} \int_{c-i \infty}^{c+i \infty} d \lambda t^{-\lambda} t^{\prime \lambda-d} D_{\lambda}(\cosh \sigma)
$$

in terms of an infinite set of Green's functions $D_{\lambda}(\cosh \sigma)$ on the anti-de Sitter slices.

In Quantum Field Theory a special role is played by the Feynman propagator which in Minkowski space is given by

$$
D_{\mathrm{F}}\left(X, X^{\prime}\right)=\Omega_{d} \frac{i}{\left(s^{2}+i \epsilon\right)^{d / 2}}
$$

with the appropriate $i \epsilon$-prescription showing that the propagator is analytic in the upper half-plane as a function of the invariant distance $s^{2}$. This translates directly into a corresponding prescription for the propagators on the de Sitter slices once we use the decomposition (5.5). We find for the Feynman-like propagator $D_{\lambda}^{\mathrm{F}}$ as function of the de-Sitter invariance distance $\sigma$ that

$$
D_{\lambda}^{\mathrm{F}}(\cos \sigma)=i D_{\lambda}(\cos \sigma-i \epsilon)
$$

This gives the correct Feynman prescription for going around the $\sigma=0$ singularity in the propagator on de Sitter spacetime.

It is now our goal to see how the Feynman propagator in Minkowski space behaves when the two points approach the boundaries at infinity of the light-cone. Suppose that one of the points approaches the future infinity of the light-cone, i.e. the sphere $S^{+}$, from the side of the region $\mathcal{D}$. In this case we have $\tau^{\prime} \rightarrow+\infty$ and the de Sitter geodesic distance behaves in this limit as

$$
z=\cos \sigma \sim \frac{1}{2} e^{\tau^{\prime}}\left(-\sinh \tau+\cosh \tau \cos \gamma\left(\theta, \theta^{\prime}\right)\right)
$$

Using the known properties (available in 21]) of the hypergeometric function when its argument approaches infinity we find that as $\tau^{\prime} \rightarrow+\infty$

$$
D_{\lambda}(z) \sim \frac{\Gamma(\lambda) \Gamma(d-2 \lambda)}{(4 \pi)^{\frac{d+1}{2}} \Gamma\left(\frac{d+1}{2}-\lambda\right)} \frac{2^{\lambda}}{(-z)^{\lambda}}+(\lambda \leftrightarrow d-\lambda)
$$

This expression contains factors $\left(\sinh \tau-\cosh \tau \cos \gamma\left(\theta, \theta^{\prime}\right)+i \epsilon\right)^{-\lambda}$. When now the second point is taken either to the future infinity $(\tau \rightarrow+\infty)$ or to the past infinity $(\tau \rightarrow-\infty)$ of 
the light-cone these factors produce non-trivial distributions on the d-sphere (see Apendix A for details)

$$
\begin{aligned}
& \lim _{\tau \rightarrow+\infty}\left(\sinh \tau-\cosh \tau \cos \gamma\left(\theta, \theta^{\prime}\right)+i \epsilon\right)^{-\lambda} \\
& =(4 \pi)^{d / 2} i^{(d-2 \lambda)} \frac{\Gamma\left(\lambda-\frac{d}{2}\right)}{\Gamma(\lambda)} e^{-(d-\lambda) \tau} \delta^{(d)}\left(\theta, \theta^{\prime}\right)+\frac{2^{\lambda} e^{-\lambda \tau}}{\left(1-\cos \gamma\left(\theta, \theta^{\prime}\right)\right)^{\lambda}}
\end{aligned}
$$

and

$$
\begin{aligned}
& \lim _{\tau \rightarrow-\infty}\left(\sinh \tau-\cosh \tau \cos \gamma\left(\theta, \theta^{\prime}\right)+i \epsilon\right)^{-\lambda} \\
& =(4 \pi)^{d / 2}(-i)^{d} \frac{\Gamma\left(\lambda-\frac{d}{2}\right)}{\Gamma(\lambda)} e^{(d-\lambda) \tau} \delta^{(d)}\left(\theta, \pi-\theta^{\prime}\right)+\frac{2^{\lambda}(-i)^{2 \lambda} e^{\lambda \tau}}{\left(1+\cos \gamma\left(\theta, \theta^{\prime}\right)\right)^{\lambda}}
\end{aligned}
$$

where $\pi-\theta$ stands for the spherical coordinates of the anti-podal point of the d-sphere, in other words $\mathbf{n}(\theta)=-\mathbf{n}(\pi-\theta)$. The expressions in (5.14) are valid up to terms of order $e^{-2 \tau}$. Using (5.14) we find the form of the Feynman propagator when both points approach the future infinity of the light-cone $\left(\tau, \tau^{\prime} \rightarrow+\infty\right)$

$$
\begin{aligned}
& \lim _{\tau, \tau^{\prime} \rightarrow+\infty} D_{\mathrm{F}}\left(X, X^{\prime}\right)=\int \frac{d \lambda}{2 \pi i} r^{-\lambda} r^{-(d-\lambda)} \\
& \left(A(\lambda) \frac{e^{-\lambda\left(\tau^{\prime}+\tau\right)}}{(1-\cos \gamma)^{\lambda}}+B(\lambda) \delta^{(d)}\left(\theta, \theta^{\prime}\right) e^{-(d-\lambda) \tau} e^{-\lambda \tau^{\prime}}+(\lambda \leftrightarrow d-\lambda)\right)
\end{aligned}
$$

where the integration over $\lambda$ goes from $d / 2-i \infty$ to $d / 2+i \infty$. A similar expression appears when $\tau, \tau^{\prime} \rightarrow-\infty$.

Notice that the first term in (5.15) looks like a conformal correlation function on one of the boundaries $\left(S_{d}^{+}\right)$of the light-cone, similar to the expression we found in the previous section. From a holographic point of view, it is a correlation function between operators associated with modes $r^{-\lambda} e^{-\lambda \tau}$ and $r^{-(d-\lambda)} e^{-\lambda \tau}$. On the other hand, we find that the correlation functions of the operators associated with the modes $r^{-\lambda} e^{-(d-\lambda) \tau}$ and $r^{-(d-\lambda)} e^{-\lambda \tau}$ produce contact terms proportional to a delta-function on the d-sphere.

When one of the points approaches the future infinity of the light-cone and another approaches the past infinity of the light-cone we find that

$$
\begin{aligned}
& \lim _{\substack{\tau^{\prime} \rightarrow+\infty \\
\tau \rightarrow-\infty}} D_{\mathrm{F}}\left(X, X^{\prime}\right)=\int \frac{d \lambda}{2 \pi i} r^{-\lambda} r^{-(d-\lambda)} \\
& \left(D(\lambda) \frac{e^{\lambda\left(\tau-\tau^{\prime}\right)}}{(1+\cos \gamma)^{\lambda}}+F(\lambda) \delta^{(d)}\left(\theta, \pi-\theta^{\prime}\right) e^{(d-\lambda) \tau} e^{-\lambda \tau^{\prime}}+(\lambda \leftrightarrow d-\lambda)\right)
\end{aligned}
$$

This also takes the form of correlation functions between conformal operators living on $S_{d}^{-}$and $S_{d}^{+}$: the correlation function diverges for antipodal points on d-sphere. This is similar to the behavior for two point functions in de Sitter space as discussed in [7, 22, 23]. The functions $A(\lambda), B(\lambda), D(\lambda)$ and $F(\lambda)$ are expressed in terms of the Gamma function as follows

$$
\begin{aligned}
& A(\lambda)=\frac{2^{\lambda} i}{4 \pi^{\frac{d}{2}+1}} \Gamma(\lambda) \Gamma\left(\frac{d}{2}-\lambda\right), \quad B(\lambda)=\frac{2^{d} i^{(d-2 \lambda+1)}}{4 \pi} \Gamma\left(\frac{d}{2}-\lambda\right) \Gamma\left(\lambda-\frac{d}{2}\right) \\
& D(\lambda)=\frac{2^{\lambda}(-i)^{(2 \lambda-1)}}{4 \pi^{\frac{d}{2}+1}} \Gamma(\lambda) \Gamma\left(\frac{d}{2}-\lambda\right), \quad F(\lambda)=\frac{2^{d}(-i)^{(d-1)}}{4 \pi} \Gamma\left(\frac{d}{2}-\lambda\right) \Gamma\left(\lambda-\frac{d}{2}\right)(5
\end{aligned}
$$


We can repeat this analysis in the case when either of the infinities of the light-cone is approached from the side of the region $\mathcal{A}$. It is useful to note that the coordinate systems (5.2) and (5.8) are related by the analytic transformation

$$
\begin{aligned}
& \mathcal{D} \rightarrow \mathcal{A}_{+}: r \rightarrow i t \quad, \quad \tau \rightarrow y-i \frac{\pi}{2} \quad, \quad \theta \rightarrow \theta \\
& \mathcal{D} \rightarrow \mathcal{A}_{-}: r \rightarrow-i t \quad, \quad \tau \rightarrow-y+i \frac{\pi}{2} \quad, \quad \theta \rightarrow \pi-\theta .
\end{aligned}
$$

Using the analytic continuation (5.18) between regions $\mathcal{A}$ and $\mathcal{D}$ one can demonstrate that the structure of the conformal correlation functions (5.15) and (5.16) is the same no matter from which side each point approaches the boundary of the light-cone. Thus, the structure of the conformal correlators emerging in (5.15) and (5.16) is an intrinsic property of the boundaries $\left(S_{d}^{-}\right.$and $\left.S_{d}^{+}\right)$themselves.

Incidentally, in de Sitter space there is a one-parameter family of vacua usually called $\alpha$-vacua, and one may wonder which vacuum state is natural once de Sitter space is embedded in Minkowski space. According to [19, the natural de Sitter vacuum to use in this context is the Bunch-Davies vacuum or the Euclidean vacuum. We have not verified whether the form of (5.16) is consistent with the two-point functions in the Euclidean vacuum as derived in [22, 23], or whether it corresponds to a different value of $\alpha$, but clearly it would be interesting to investigate this.

\section{$5.2 \quad$ In- and out-fields}

The standard decomposition of asymptotic fields in terms of plane waves is

$$
\phi\left(X_{0}, \mathbf{X}\right)=\int d \mathbf{k}\left(a(\mathbf{k}) f_{k}\left(X_{0}, \mathbf{X}\right)+a^{\dagger}(\mathbf{k}) f_{k}^{*}\left(X_{0}, \mathbf{X}\right)\right)
$$

where the functions

$$
f_{k}\left(X_{0}, \mathbf{X}\right)=\frac{1}{\left[(2 \pi)^{d+1} 2 k\right]^{1 / 2}} e^{-i\left(k X_{0}-\mathbf{k} \mathbf{X}\right)}
$$

form a basis of (positive frequency) asymptotic fields. The $(\mathrm{d}+1)$-vector $\mathbf{k}$ is parametrized by its length $k=\sqrt{\mathbf{k k}}$ and angles $\left\{\theta_{k}\right\}$ which determine the direction of the vector in

momentum space. Hence we have $\mathbf{k}=k \mathbf{n}\left(\theta_{k}\right)$, where $\mathbf{n}\left(\theta_{k}\right)$ is unit vector providing the direction. The creation and annihilation operators satisfy the usual algebra

$$
\left[a(\mathbf{k}), a^{\dagger}\left(\mathbf{k}^{\prime}\right)\right]=\delta^{(d+1)}\left(\mathbf{k}, \mathbf{k}^{\prime}\right)=\frac{1}{k^{d}} \delta\left(k-k^{\prime}\right) \delta^{(d)}\left(\theta_{k}, \theta_{k^{\prime}}\right),
$$

where $\delta^{(d)}\left(\theta_{k}, \theta_{k^{\prime}}\right)$ is the delta-function on the d-sphere with respect to the standard measure $d \mu\left(\theta_{k}\right)$.

In the region $\mathcal{A}$ the coordinates $\left(X_{0}, \mathbf{X}\right)$ are given by (5.8) so that the argument in the exponent in (5.21) can be written in the form

$$
\left(k X_{0}-\mathbf{k X}\right)=k t\left(\cosh y-\sinh y \cos \gamma\left(\theta, \theta_{k}\right)\right) .
$$

Using this expression and formula (3.7) we obtain a representation of the plane waves

$$
e^{ \pm i\left(k X_{0}-\mathbf{k X}\right)}=\frac{1}{2 \pi i} \int_{c-i \infty}^{c+i \infty} d \lambda \frac{( \pm i)^{\lambda} \Gamma(\lambda) k^{-\lambda}(t \pm i \epsilon)^{-\lambda}}{\left(\cosh y-\sinh y \cos \gamma\left(\theta, \theta_{k}\right)\right)^{\lambda}}
$$


where the $i \epsilon$-prescription determines the way to go around the $t=0$ singularity. With the help of this representation we can obtain an explicit decomposition of the asymptotic field (5.19) in terms of data on the boundary of the light-cone.

Consider the region $\mathcal{A}_{+}$in which the coordinate $t>0$. With the help of (15.22) the asymptotic field (5.19) has the form

$$
\phi(t, y, \theta)=\frac{1}{2 \pi i} \int_{d / 2-i \infty}^{d / 2+i \infty} d \lambda t^{-\lambda} \int_{S_{d}^{+}} d \mu\left(\theta_{k}\right) G_{(\lambda)}\left(y, \theta, \theta_{k}\right) \phi^{(d-\lambda)}\left(\theta_{k}\right)
$$

where

$$
G_{(\lambda)}\left(y, \theta, \theta_{k}\right)=\frac{g(\lambda)}{\left(\cosh y-\sinh y \cos \left(\theta, \theta_{k}\right)\right)^{\lambda}}
$$

is the bulk-boundary propagator on Euclidean anti-de Sitter introduced in (4.5). The family of functions (parametrized by $\lambda$ )

$$
\phi^{(d-\lambda)}\left(\theta_{k}\right)=\frac{2^{\frac{d}{2}} \Gamma\left(\lambda-\frac{d}{2}\right)}{2 \pi^{\frac{1}{2}}} \int_{0}^{\infty} d k k^{\left(d-\lambda-\frac{1}{2}\right)}\left(e^{-i \frac{\pi}{2} \lambda} a\left(k, \theta_{k}\right)+e^{i \frac{\pi}{2} \lambda} a^{\dagger}\left(k, \theta_{k}\right)\right)
$$

can be thought of as the boundary data on the boundary $S_{d}^{+}$of the future light-cone. Equation (5.23) thus gives us a solution to a quite peculiar boundary problem and shows how the field in $(\mathrm{d}+2)$-dimensional space-time can be reconstructed from data on a codimension two surface lying at the boundary at infinity of the light-cone.

The algebra (5.21) induces the algebra of the operators (5.25) and we obtain for their vacuum expectation values

$$
\begin{aligned}
& <0\left|\phi^{(d-\lambda)}\left(\theta_{k}\right) \phi^{\left(d-\lambda^{\prime}\right)}\left(\theta_{k}^{\prime}\right)\right| 0> \\
& =\frac{2^{d}}{4 \pi} \Gamma\left(\lambda-\frac{d}{2}\right) \Gamma\left(\lambda^{\prime}-\frac{d}{2}\right)(-i)^{\lambda} i^{\lambda^{\prime}} 2 \pi i \delta\left(\lambda+\lambda^{\prime}-d\right) \delta^{(d)}\left(\theta_{k}, \theta_{k}^{\prime}\right) .
\end{aligned}
$$

Using this we can compute the vacuum expectation value of the asymptotic fields (5.19) and we find that

$$
\begin{aligned}
& <0\left|\left\{\phi(t, y, \theta), \phi\left(t^{\prime}, y^{\prime}, \theta^{\prime}\right)\right\}\right| 0> \\
& =\frac{1}{2 \pi i} \int_{d / 2-i \infty}^{d / 2+i \infty} d \lambda t^{-\lambda} t^{\prime-(d-\lambda)} C(\lambda) \int_{S_{d}^{+}} d \mu\left(\theta_{k}\right) G_{(\lambda)}\left(y, \theta_{k}, \theta\right) G_{(d-\lambda)}\left(y^{\prime}, \theta_{k}, \theta^{\prime}\right), \\
& C(\lambda)=\frac{2^{d}}{2 \pi} \Gamma\left(\lambda-\frac{d}{2}\right) \Gamma\left(\frac{d}{2}-\lambda\right) \cos \left(\frac{\pi}{2}(d-2 \lambda)\right) .
\end{aligned}
$$

Equation (5.27) is another representation for the Hadamard function (5.11). Comparing these two expressions we find a representation for the anti-de Sitter bulk-bulk propagator $D_{\lambda}(\cosh \sigma)$ in terms of the bulk-boundary propagator (5.24). This representation is rather natural from the point of view of anti-de Sitter space.

In the region $\mathcal{A}_{-}$where the coordinate $t<0$ there exists a representation for the asymptotic field which is similar to (5.23): one should just switch signs appropriately according to the $i \epsilon$-prescription in (5.22).

In region $\mathcal{D}$ foliated by de Sitter slices the coordinates $\left(X_{0}, \mathbf{X}\right)$ are given by (5.2) and we have that

$$
\left(k X_{0}-\mathbf{k X}\right)=k r\left(\sinh \tau-\cosh \tau \cos \gamma\left(\theta, \theta_{k}\right)\right)
$$


The expression for the plane waves then reads

$$
e^{ \pm i\left(k X_{0}-\mathbf{k X}\right)}=\frac{1}{2 \pi i} \int_{c-i \infty}^{c+i \infty} d \lambda \frac{( \pm i)^{\lambda} \Gamma(\lambda) k^{-\lambda} r^{-\lambda}}{\left(\sinh \tau-\cosh \tau \cos \gamma\left(\theta, \theta_{k}\right) \pm i \epsilon\right)^{\lambda}}
$$

The kernel appearing in (5.28) is singular and ambiguous. The $i \epsilon$ in (5.28) provides the appropriate way to deal with the singularity in the kernel. The asymptotic behavior of such kernels is given by (5.13) and (5.14). Using those asymptotics we define the bulk to boundary propagator near the boundary at future infinity of the light-cone as follows

$$
G_{\lambda}^{\text {out }}\left(\tau, \theta, \theta^{\prime}\right)=\frac{g(\lambda)(-i)^{d-2 \lambda}}{\left(\sinh \tau-\cosh \tau \cos \gamma\left(\theta, \theta^{\prime}\right)+i \epsilon\right)^{\lambda}}
$$

The bulk-boundary propagator near the boundary at past infinity is defined as

$$
G_{\lambda}^{\text {in }}\left(\tau, \theta, \theta^{\prime}\right)=\frac{g(\lambda)(-i)^{d}}{\left(\sinh \tau+\cosh \tau \cos \gamma\left(\theta, \theta^{\prime}\right)-i \epsilon\right)^{\lambda}} .
$$

We should note that the $\epsilon$-prescription used to define the decomposition of the plane waves (see (5.22) and (5.28) ) is in general not analytic under the transformation (5.18) between regions $\mathcal{A}$ and $\mathcal{D}$. We find that modes $e^{-i k X}$ with $k^{0}>0$ are analytic across the future light-cone while modes $e^{i k X}$ with $k^{0}>0$ are analytic across the past light-cone.

\subsection{Conformal operators}

The analysis of the previous subsection suggests that one can define the conformal operators as associated with the asymptotic (possibly, interacting in the bulk) in- and outquantum fields. For the out-field the representation is

$$
\lim _{\tau \rightarrow+\infty} \phi(r, \tau, \theta)=\frac{1}{2 \pi i} \int_{\frac{d}{2}-i \infty}^{\frac{d}{2}+i \infty} d \lambda\left(\varphi_{\lambda}^{<}(r, \tau){ }_{\text {out }} \mathcal{O}_{\lambda}^{<}(\theta)+\varphi_{\lambda}^{>}(r, \tau){ }_{\text {out }} \mathcal{O}_{\lambda}^{>}(\theta)\right)
$$

For the in-field we have a similar representation in terms of the in-operators

$$
\lim _{\tau \rightarrow-\infty} \phi(r, \tau, \theta)=\frac{1}{2 \pi i} \int_{\frac{d}{2}-i \infty}^{\frac{d}{2}+i \infty} d \lambda\left(\chi_{\lambda}^{>}(r, \tau){ }_{\text {in }} \mathcal{O}_{\lambda}^{>}(\theta)+\chi_{\lambda}^{<}(r, \tau){ }_{\text {in }} \mathcal{O}_{\lambda}^{<}(\theta)\right)
$$

The asymptotic modes which are right-moving as a function of $\lambda$ on the $(\log r, \tau)$-plane are labelled by the subscript $>$ while the left-moving modes are labelled by $<$. The functions

$$
\varphi_{\lambda}^{<}(r, \tau)=N_{\lambda}^{-1} r^{-\lambda} e^{-\lambda \tau}, \quad \varphi_{\lambda}^{>}(r, \tau)=N_{\lambda}^{-1} i^{\lambda-d} r^{-\lambda} e^{-(d-\lambda) \tau}
$$

form the basis of out-modes and the functions

$$
\chi_{\lambda}^{>}(r, \tau)=N_{\lambda}^{-1} r^{-\lambda} e^{\lambda \tau}, \quad \chi_{\lambda}^{<}(r, \tau)=N_{\lambda}^{-1} i^{\lambda-d} r^{-\lambda} e^{(d-\lambda) \tau}
$$

form the basis of the in-modes on the $(\log r, \tau)$-plane. In the region $\mathcal{A}$ the respective modes are those obtained from (5.33) and (5.34) by means of the analytic continuation (5.18). The conformal operators so defined are associated with the boundary of the lightcone at infinity and are independent of the side $(\mathcal{A}$ or $\mathcal{D})$ from which the boundary is approached. 
Since $\tau$ plays the role of time in the de Sitter foliation of Minkowski space it can be used to define positive and negative frequencies. Representing $\lambda=\frac{d}{2}+i \alpha$ where $\alpha$ is a real parameter we then find that the positive frequency modes are $\chi_{(-\alpha)}^{>}, \chi_{(\alpha)}^{<}, \varphi_{(\alpha)}^{<}$and $\varphi_{(-\alpha)}^{>}$, all with $\alpha$ positive. Inversion of the sign of $\alpha$ (or equivalently taking the complex conjugate) then gives the negative-frequency modes. The constant $N_{\lambda}$ is given by

$$
N_{\lambda}=2^{-\frac{d}{2}} \sqrt{2 \lambda-d}
$$

The positive frequency modes then are normalized as

$$
\left(f_{\lambda}, f_{\lambda^{\prime}}\right)=2 \pi i \delta\left(\lambda-\lambda^{\prime}\right)
$$

where $f_{\lambda}$ stands for any of the positive frequency modes we defined above. The inner product is given by the usual Klein-Gordon expression

$$
(f, g)=(\cosh \tau)^{d} \int_{0}^{\infty} d r r^{d-1} f^{*} \overleftrightarrow{i \partial_{\tau}} g
$$

where $\tau \rightarrow+\infty$ for the out-operators and $\tau \rightarrow-\infty$ for in-operators.

For the operators defined in (5.31) and (5.32) we have that $\mathcal{O}_{-\alpha}^{<}=\left[\mathcal{O}_{\alpha}^{<}\right]^{*}$ and $\mathcal{O}_{-\alpha}^{>}=$ $\left[\mathcal{O}_{\alpha}^{>}\right]^{*}$. This implies that the in- and out-operators $\mathcal{O}_{\alpha}^{<}$and $\mathcal{O}_{-\alpha}^{>}$couple to positivefrequency modes while $\mathcal{O}_{\alpha}^{>}$and $\mathcal{O}_{-\alpha}^{<}$couple to negative-frequency modes. This definition of the positive and negative frequencies is different from the definition based on the global time coordinate $X_{0}$. The difference between two is reminiscent of the well-known relation between the Rindler and Minkowski definitions of time in Rindler spacetime. The corresponding modes are related by a Bogoliubov transform. As is seen from (5.28) the modes which have positive frequency in the sense of the time-like vector $\partial_{X_{0}}$ decompose onto both negative and positive frequency modes in the sense of de Sitter time vector $\partial_{\tau}$. It would be interesting to understand better the implications of this observation. Note also that the definition of the conformal operators given in (5.31) and (5.32) is reminiscent of the analysis of section 4 .

The operation inverse to the spectral integration in (5.31), (5.32) is the direct Mellin transform

$$
\phi_{\lambda}(\tau, \theta)=\int_{0}^{\infty} d r r^{\lambda-1} \phi(r, \tau, \theta)
$$

so that $\phi_{\lambda}(\tau, \theta)$ can be thought of as a field living on de Sitter space with coordinates $(\tau, \theta)$. On the other hand the kernels $G_{\lambda}^{\text {in }}\left(\tau, \theta, \theta^{\prime}\right)$ and $G_{\lambda}^{\text {out }}\left(\tau, \theta, \theta^{\prime}\right)$ given in (5.29) and (5.30) can be used to project ${ }^{\|}$the field $\phi_{\lambda}(\tau, \theta)$ onto modes asymptotic to $e^{ \pm \lambda \tau}$ and $e^{ \pm(d-\lambda) \tau}$. These two operations can be used to derive the insertion of the in- and out-conformal operators in terms of the (in general interacting) bulk Feynman Green's function in the form of an integral over a hypersurface of constant $\tau$. For the out-operators it is the surface at $\tau=+\infty$ while for the in-operators we should take the surface at $\tau=-\infty$. The insertion prescriptions are

$$
\begin{aligned}
& <0\left|. .{ }_{\text {in }} \mathcal{O}_{\lambda}^{<}(\theta) . .\right| 0>= \\
& \lim _{\tau \rightarrow-\infty} \int_{0}^{\infty} d r r^{\lambda-1} N_{\lambda}^{-1} i^{d-\lambda} \int_{S_{d}^{-}} d \mu\left(\theta^{\prime}\right)(\cosh \tau)^{d} D_{\mathrm{F}}\left(. .,\left(r, \tau, \theta^{\prime}\right), . .\right) \overleftrightarrow{\partial_{\tau}} G_{d-\lambda}^{\text {in }}\left(\tau, \theta, \theta^{\prime}\right)
\end{aligned}
$$

"There are two terms in the asymptotic expansion of $G_{\lambda}^{\text {in (out) }}\left(\tau, \theta, \theta^{\prime}\right)$, see (5.13) and (5.14). In order for the term with the delta-function to dominate we take $R e \lambda=\frac{d}{2}+\epsilon$ and take $\epsilon$ to zero afterwards. 
and

$$
\begin{aligned}
& <0\left|. .{ }_{\text {in }} \mathcal{O}_{\lambda}^{>}(\theta) . .\right| 0>= \\
& \lim _{\tau \rightarrow-\infty} \int_{0}^{\infty} d r r^{\lambda-1} N_{\lambda}^{-1} \int_{S_{d}^{-}} d \mu\left(\theta^{\prime}\right)(\cosh \tau)^{d} D_{\mathrm{F}}\left(. .,\left(r, \tau, \theta^{\prime}\right), . .\right) \overleftrightarrow{\partial_{\tau}} G_{\lambda}^{\text {in }}\left(\tau, \theta, \theta^{\prime}\right)
\end{aligned}
$$

for the in-operators and

$$
\begin{aligned}
& <0 \mid . . \text { out } \mathcal{O}_{\lambda}^{<}(\theta) . . \mid 0>= \\
& \lim _{\tau \rightarrow+\infty} \int_{0}^{\infty} d r r^{\lambda-1} N_{\lambda}^{-1} \int_{S_{d}^{+}} d \mu\left(\theta^{\prime}\right)(\cosh \tau)^{d} G_{\lambda}^{\text {out }}\left(\tau, \theta, \theta^{\prime}\right) \overleftrightarrow{\partial_{\tau}} D_{\mathrm{F}}\left(. .,\left(r, \tau, \theta^{\prime}\right), . .\right)
\end{aligned}
$$

and

$$
\begin{aligned}
& <0 \mid . . \text { out } \mathcal{O}_{\lambda}^{>}(\theta) . . \mid 0>= \\
& \lim _{\tau \rightarrow+\infty} \int_{0}^{\infty} d r r^{\lambda-1} N_{\lambda}^{-1} i^{d-\lambda} \int_{S_{d}^{+}} d \mu\left(\theta^{\prime}\right)(\cosh \tau)^{d} G_{d-\lambda}^{\text {out }}\left(\tau, \theta, \theta^{\prime}\right) \overleftrightarrow{\partial_{\tau}} D_{\mathrm{F}}\left(. .,\left(r, \tau, \theta^{\prime}\right), . .\right)
\end{aligned}
$$

for the out-operators. Near the boundary $\left(S^{+}\right.$or $\left.S^{-}\right)$of the light-cone the coordinate $r$ plays the role of a coordinate enumerating the de Sitter slices ending at the surface $S^{+}$ (or $S^{-}$). Thus, in the above formulas an insertion effectively takes place at each of the de Sitter slices separately, after which one sums over all de Sitter slices labelled by $r$.

These expressions are very similar to the ones introduced in [7, 23] for the insertion of boundary conformal operators in the case of de Sitter space. The field $\phi_{\lambda}(\tau, \theta)$ obtained from $\phi(r, \tau, \theta)$ by the Mellin transform in the variable $r$ describes a field on de Sitter space with mass equal $\lambda(d-\lambda)$, so perhaps this is not too surprising. Nevertheless, it is interesting to observe that computing the on-shell action (as we attempted in section 4) is not very satisfactory, whereas the S-matrix approach in this section gives a concise proposal for a Minkowski/CFT duality as in (5.41), just as in de Sitter space.

Inside the light-cone, in the region $\mathcal{A}$, the insertions can be defined in a similar way as in (5.38), (5.39), (5.40), (5.41). The appropriate surface is then the boundary at $y=+\infty$ of each anti-de Sitter slice and one has to integrate over contributions of all such slices, which translates into an integral over negative $t$ for the in-operators and over positive $t$ for the out-operators. On an anti-de Sitter slice we could use the definition of the conformal correlation functions built out of bulk-boundary propagators and truncated n-point Feynman Green's function on the slice, as familiar from AdS/CFT, see [5], [24]. Notice that even on the Anti-de Sitter slices computing an on-shell action is not the right approach, because of the complex values of the conformal weights, and therefore even on the AdS slices we should resort to an S-matrix type approach like the one discussed in [25].

The integration over $t$ adds up the contributions of all AdS slices. The explicit form of the insertions in region $\mathcal{A}$ can be obtained by applying the analytic transformation (5.18) to (5.38)-(15.41).

It appears that correlation functions defined in this way do not depend on the choice of region (inside or outside the light-cone) which is used to define the insertions and are therefore an intrinsic property of the boundary of the light-cone. The 2-point correlation functions between out- and in-operators are now found to be equal to

$$
<\left.0\right|_{\text {out }} \mathcal{O}_{\lambda_{1}}^{<}\left(\theta_{1}\right){ }_{\text {in }} \mathcal{O}_{\lambda_{2}}^{>}\left(\theta_{2}\right) \mid 0>=2 \pi i \delta\left(\lambda_{1}+\lambda_{2}-d\right) \delta^{(d)}\left(\theta_{1}, \pi-\theta_{2}\right) i^{d} N_{\lambda_{1}} N_{\lambda_{2}} F\left(\lambda_{2}\right)(5.41)
$$




$$
\begin{gathered}
<\left.0\right|_{\text {out }} \mathcal{O}_{\lambda_{1}}^{>}\left(\theta_{1}\right){ }_{\text {in }} \mathcal{O}_{\lambda_{2}}^{<}\left(\theta_{2}\right) \mid 0>=2 \pi i \delta\left(\lambda_{1}+\lambda_{2}-d\right) \delta^{(d)}\left(\theta_{1}, \pi-\theta_{2}\right) i^{d} N_{\lambda_{1}} N_{\lambda_{2}} F\left(\lambda_{1}\right)(5 \\
<\left.0\right|_{\text {out }} \mathcal{O}_{\lambda_{1}}^{>}\left(\theta_{1}\right){ }_{\text {in }} \mathcal{O}_{\lambda_{2}}^{>}\left(\theta_{2}\right) \mid 0>=2 \pi i \delta\left(\lambda_{1}+\lambda_{2}-d\right) \frac{i^{d-\lambda_{2}} N_{\lambda_{1}} N_{\lambda_{2}} D\left(\lambda_{2}\right)}{\left(1+\cos \gamma\left(\theta_{1}, \theta_{2}\right)\right)^{\lambda_{2}}} \\
<\left.0\right|_{\text {out }} \mathcal{O}_{\lambda_{1}}^{<}\left(\theta_{1}\right){ }_{\text {in }} \mathcal{O}_{\lambda_{2}}^{<}\left(\theta_{2}\right) \mid 0>=2 \pi i \delta\left(\lambda_{1}+\lambda_{2}-d\right) \frac{i^{d-\lambda_{1}} N_{\lambda_{1}} N_{\lambda_{2}} D\left(\lambda_{1}\right)}{\left(1+\cos \gamma\left(\theta_{1}, \theta_{2}\right)\right)^{\lambda_{1}}}
\end{gathered}
$$

Thus, the set of CFT operators splits into two groups: operators $\mathcal{O}^{>}$representing the right-moving modes and operators $\mathcal{O}^{<}$representing the left-moving modes. The correlation functions of operators of the same group take a standard CFT form (5.43), (5.44) on a d-sphere. On the other hand, operators from different groups produce contact terms in the correlators (5.41), (5.42). Note that contact type correlation functions are also consistent with conformal invariance.

\subsection{S-matrix}

It is well-known that in Minkowski space the S-matrix of interacting quantum fields can be reconstructed from bulk correlation functions via the so-called LSZ construction. A natural question arises whether it is possible to reproduce the S-matrix by means of only the light-cone correlation functions of conformal operators introduced in section 5.3. That this may be the case is suggested by the analysis made in de Sitter space [23] where it was shown that the de Sitter analog of an S-matrix indeed can be presented in terms of CFT correlation functions between operators living at the infinite past and future of de Sitter space. In anti-de Sitter a similar construction was introduced in [25].

In order to analyze this possibility we consider the one-particle amplitude whose LSZ form is given by a combination of integrating over two surfaces, one lying in the infinite past and another in the infinite future of Minkowski space [26],

$$
\begin{aligned}
& <p \mid q>=\int \frac{d V(X)}{\sqrt{Z}} \int \frac{d V(Y)}{\sqrt{Z}} f_{q}(X) \overrightarrow{\nabla^{2}}{ }_{X} D_{\mathrm{F}}(Y, X) \overleftrightarrow{\nabla_{Y}^{2}} f_{p}^{*}(Y) \\
& =-\int_{Y_{0} \rightarrow+\infty} d \sigma(Y) \int_{X_{0} \rightarrow-\infty} d \sigma(X) n_{x}^{\mu} f_{q}(X) \overleftrightarrow{\partial_{\mu}} D_{\mathrm{F}}(Y, X) \overleftrightarrow{\partial_{\nu}} f_{p}^{*}(Y) n_{y}^{\nu},
\end{aligned}
$$

where $Z$ is the standard normalization of the wave function and $n_{x}^{\mu}$ is the normal vector to the hypersurface in the infinite past while $n_{y}^{\mu}$ is the normal to the hypersurface in the infinite future. The functions $f_{k}(X)$ are defined in (5.20) and form a basis of the set of asymptotic fields. We recall that in momentum space the asymptotic spatial momentum $\mathbf{k}$ is determined by its length $k$ and angles $\left\{\theta_{k}\right\}$ on a d-sphere so that $\mathbf{k}=k \mathbf{n}\left(\theta_{k}\right)$.

For our purposes it is convenient to choose the asymptotic hypersurfaces in a different way. Namely, we define the asymptotic future and past hypersurfaces by $\Sigma_{\text {out }}=\{\mathcal{A}: y=$ const $\rightarrow \infty(t>0)\} \cup\{\mathcal{D}: \tau=$ const $\rightarrow \infty\}$ and $\Sigma_{\text {in }}=\{\mathcal{A}: y=$ const $\rightarrow \infty(t<$ $0)\} \cup\{\mathcal{D}: \tau=$ const $\rightarrow-\infty\}$. We see that these hypersurfaces are defined in the vicinity of the boundary of the light-cone and consist of two components: one in region $\mathcal{A}$ and another in region $\mathcal{D}$. As we show in Appendix $\mathrm{B}$ the plane waves $f_{q}(X)$ have the standard normalization with respect to the Klein-Gordon inner product defined at these hypersurfaces. Thus, $\Sigma_{\text {out }}$ and $\Sigma_{\text {in }}$ can be used to define asymptotic states in the 


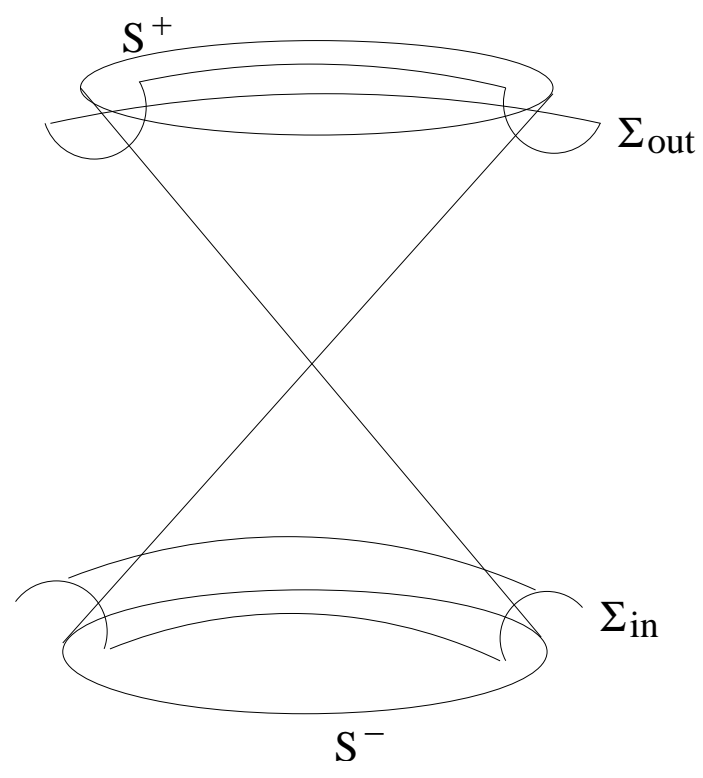

Fig. 2: The asymptotic hypersurfaces $\Sigma_{\text {in }}$ and $\Sigma_{\text {out }}$ are defined in a small vicinity of the past and future infinity of the light-cone respectively.

same fashion as the usual constant $X_{0}$ surfaces. With this definitions and these choices of asymptotic surfaces the LSZ form of the one-particle amplitude (5.45) is modified into

$$
<p \mid q>=\int_{\Sigma_{\text {out }}} \frac{d \sigma}{\sqrt{Z}} \int_{\Sigma_{\text {in }}} \frac{d \sigma^{\prime}}{\sqrt{Z}} f_{p}^{*}(X) \overleftrightarrow{\partial_{n}} D_{F}\left(X, X^{\prime}\right) \overleftrightarrow{\partial_{n}^{\prime}} f_{q}\left(X^{\prime}\right)
$$

The asymptotic functions $f_{q}(X)$ and $f_{p}^{*}(Y)$ can be decomposed according to (5.28) in the region $\mathcal{D}$ and (5.22) in the region $\mathcal{A}$. Inserting this decomposition into (5.46) and after some manipulations we recognize the two-point functions defined in section 5.3. Consequently, the one-particle amplitude takes the form

$$
\begin{aligned}
& <p \mid q>=\frac{\sqrt{p q}}{(2 \pi i)^{2}} \int \frac{d \lambda_{1}}{\sqrt{Z}} \int \frac{d \lambda_{2}}{\sqrt{Z}} p^{-\lambda_{1}} q^{-\lambda_{2}} H\left(\lambda_{1}\right) H\left(\lambda_{2}\right) \\
& <\left.0\right|_{\text {out }} \mathcal{O}_{d-\lambda_{1}}^{<}\left(\theta_{p}\right){ }_{\text {in }} \mathcal{O}_{d-\lambda_{2}}^{>}\left(\pi-\theta_{q}\right) \mid 0>,
\end{aligned}
$$

where

$$
H(\lambda)=\frac{2^{\frac{d}{2}}}{\sqrt{\pi}} i \Gamma\left(\lambda-\frac{d}{2}\right) \sin \frac{\pi}{2}(d-2 \lambda)
$$

and each $\lambda$-integration is from $\frac{d}{2}-i \infty$ to $\frac{d}{2}+i \infty$. The function $f_{p}^{*}(X)$, once expressed in terms of conformal operators, is not analytic across the future light-cone. The jump is $i^{d-2 \lambda}-i^{2 \lambda-d}$, which explains the appearance of $\sin \frac{\pi}{2}(d-2 \lambda)$ in the function $H(\lambda)$. The same is true for the function $f_{q}(X)$ near the boundary at infinity of the past light-cone. For free fields the light-cone 2-point function in (5.47) is given by (5.41) and after the integration over $\lambda_{1}$ and $\lambda_{2}$ the usual expression

$$
<p \mid q>=p^{-d} \delta(p-q) \delta^{(d)}\left(\theta_{p}, \theta_{q}\right)
$$

is reproduced. Although this calculation was performed for a free field interaction is not expected to change the form of the conformal correlation function in the amplitude (5.47), 
but only its normalization. Therefore the angle part of the amplitude comes out in the same way as in (5.48) while the $p$-dependent pre-factor can be modified. Note that this is also consistent with the restrictions imposed by bulk Lorentz invariance on the structure of the one-particle amplitude.

The generalization of (5.47) to many-particle amplitudes is straightforward. It is important to note that the amplitude (5.47) is given in terms of the contact type correlation functions of operators introduced in section 5.3.

In the construction of the scattering amplitudes (5.45) and (5.47) the positive and negative frequency modes of the asymptotic fields are defined with respect to the vector field $\partial_{X_{0}}$ which is a global time-like vector in Minkowski space. Instead we could have chosen to use the time $\tau$ on a de Sitter slice to define the positive and negative frequency modes. The basis of asymptotic fields then is given by the functions (5.34) and (5.33) These modes can be used to form incoming $(f)$ and outgoing $(g)$ wavepackets as

$$
\begin{aligned}
f^{>}(r, \tau, \theta) & =\frac{1}{2 \pi i} \int d \lambda \chi_{\lambda}^{>}(r, \tau) f_{\lambda}^{>}(\theta), \quad f^{<}(r, \tau, \theta)=\frac{1}{2 \pi i} \int d \lambda \chi_{\lambda}^{<}(r, \tau) f_{\lambda}^{<}(\theta), \\
g^{>}(r, \tau, \theta) & =\frac{1}{2 \pi i} \int d \lambda \varphi_{\lambda}^{>}(r, \tau) g_{\lambda}^{>}(\theta), \quad g^{<}(r, \tau, \theta)=\frac{1}{2 \pi i} \int d \lambda \varphi_{\lambda}^{<}(r, \tau) g_{\lambda}^{<}(\theta),
\end{aligned}
$$

where $f_{\lambda}^{>}(\theta)\left(f_{\lambda}^{<}(\theta)\right)$ and $g_{\lambda}^{>}(\theta)\left(g_{\lambda}^{<}(\theta)\right)$ are smooth functions on the spheres $S_{d}^{-}$and $S_{d}^{+}$ respectively. In order to make sure that only positive frequency modes contribute to the wavepacket we demand that (provided that $\left.\lambda=\frac{d}{2}+i \alpha\right) f_{\alpha}^{>}(\theta)=0$ and $f_{-\alpha}^{<}(\theta)=0$ for $\alpha>0$, with similar conditions on the envelope functions in the outgoing wavepacket. The operators $b_{f}^{>(<)}$and $b_{g}^{>(<)}$corresponding to these wavepackets are recovered from the asymptotic free field (5.31) (or (5.32)) by the standard formulas

$$
\begin{aligned}
& b_{f}^{>}=\int_{0}^{\infty} d r r^{d-1} \int_{S_{d}^{-}} d \mu(\theta)(\cosh \tau)^{d}\left[f^{>}(r, \tau, \theta)\right]^{*} i \overleftrightarrow{\partial_{\tau}} \phi_{\text {in }}(r, \tau, \theta) \\
& b_{g}^{>}=\int_{0}^{\infty} d r r^{d-1} \int_{S_{d}^{+}} d \mu(\theta)(\cosh \tau)^{d}\left[g^{>}(r, \tau, \theta)\right]^{*} i \overleftrightarrow{\partial_{\tau}} \phi_{\text {out }}(r, \tau, \theta)
\end{aligned}
$$

with similar expressions for the left-moving modes. The S-matrix elements for an incoming wavepacket $f^{>}$and an outcoming wavepacket $g^{>}$are defined by

$$
S\left[g^{>}, f^{>}\right]=<0\left|b_{g}^{>} b_{f}^{>\dagger}\right| 0>
$$

These matrix elements can be re-written in terms of 2-point correlation functions of the conformal operators $\mathcal{O}^{>}$as

$$
S\left[g^{>}, f^{>}\right]=\frac{1}{(2 \pi i)^{2}} \int \frac{d \lambda}{\sqrt{Z}} \int \frac{d \lambda^{\prime}}{\sqrt{Z}} \int_{S_{d}^{+}}\left[g_{\lambda}^{>}(\theta)\right]^{*} \int_{S_{d}^{-}} f_{\lambda^{\prime}}^{>}\left(\theta^{\prime}\right)<\left.0\right|_{\text {out }} \mathcal{O}_{d-\lambda}^{>}(\theta)_{\text {in }} \mathcal{O}_{\lambda^{\prime}}^{>}\left(\theta^{\prime}\right) \mid 0>
$$

A similar expression exists for the matrix element $S\left[g^{<}, f^{<}\right]$. This formula relates the matrix elements of the S-matrix with the light-cone conformal correlation functions of operators living on the asymptotic d-spheres. A generalization of this formula to manyparticle amplitudes is straightforward. Our analysis suggests that the S-matrix approach to Quantum Field Theory in Minkowski space can be re-formulated entirely in terms of the correlation functions of conformal operators living on the boundaries of the lightcone. This opens the possibility to find a truly holographic representation of the physics in Minkowski space. For such a holographic representation we should also consider, obviously, gravity in Minkowski space, to which we turn next. 


\section{Asymptotic light-cone structure and conformal symmetry}

\subsection{Asymptotic Minkowski Spaces}

In this section we consider a more general class of metrics which are not globally Minkowski but approach flat space in a suitable asymptotic sense. In particular, we are interested in the asymptotic light-cone structure of the metric and the asymptotic symmetries associated to this structure.

First, we generalize the Minkowski metric studied in the previous sections. It should approach the Minkowski metric asymptotically in such a way that at infinity a slicing with constant curvature surfaces is appropriate. For concreteness we consider only the region $\mathcal{A}_{-}$. We start with the following asymptotic form of the $(\mathrm{d}+2)$-metric

$$
d s^{2}=-d t^{2}+t^{2}\left(\frac{d \rho^{2}}{4 \rho^{2}}\left(1+t^{-1} \sigma(t, \theta, \rho)\right)+\frac{1}{\rho} g_{i j}(t, \theta, \rho) d \theta^{i} d \theta^{j}+2 t^{-1} A_{i}(t, \theta, \rho) d \theta^{i} d \rho\right)
$$

where normal coordinates, in which the components $G_{t \rho}$ and $G_{t i}$ vanish, have been used. The metric in parenthesis in (6.1) is the $(d+1)$-metric on an Euclidean slice of constant $t$. Provided that the $(d+2)$-metric is Ricci flat, the $t=$ const slice of the light-cone metric (6.1) should for large $t$ approach a solution of the Einstein equations with negative cosmological constant. Therefore, the choice of the metric (6.1) to the leading order in $t$ is motivated by the general form of the negative constant curvature metric studied in the context of the AdS/CFT correspondence [27]. The conformal boundary of a $t=$ const slice is at $\rho=0$. For large $t$ each function in (6.1) is assumed to have an expansion in powers of $1 / t$ as well as in powers of $\rho^{1 / 2}$. For instance, the metric $g_{i j}(t, \theta, \rho)$ can be expanded as

$$
g_{i j}(t, \theta, \rho)=g_{i j}^{(0)}(\theta, \rho)+t^{-1} g_{i j}^{(1)}(\theta, \rho)+\ldots,
$$

which could also include logarithmic terms of the form $t^{-n}(\ln t)^{-m}$ and each term in the $t$-expansion should be also expanded in terms of $\rho^{1 / 2}$, e.g.

$$
g_{i j}^{(n)}(\theta, \rho)=g_{i j}^{(n, 0)}(\theta)+g_{i j}^{(n, 1)}(\theta) \rho^{1 / 2}+\ldots,
$$

where $g_{i j}^{(0,0)}(\theta)$ is to be interpreted as the metric on the boundary $\Sigma_{d}^{-}$of the past lightcone. This boundary metric should be the same for all $t=$ const slices. Expanding first in powers of $\rho$ this condition says that the constant term in the expansion should not be a function of $t$, i.e. $g_{i j}(t, \theta, \rho)=g_{i j}^{(0,0)}(\theta)+O\left(\rho^{1 / 2}\right)$. For large $t$ this gives a restriction on

the coefficients $g_{i j}^{(n, 0)}$, namely only the one with $n=0$ should be non-vanishing. We do the holography along the $\rho$-direction so that no additional expansion in $t$-variable should be necessary. It is however technically more convenient to work with functions which have certain expansion in powers of $1 / t$. A bonus we get is extra diffeomorphisms which generate transformations in the $t$ directions and change the metric to $1 / t$ order. The BMS group will be a part of these diffeomorphisms.

When we analyze the bulk diffeomorphisms that preserve the asymptotic form (6.1) we find that there is a particular $t$-independent diffeomorphism generated by

$$
\xi^{t}=0 \quad, \quad \xi^{\rho}=\gamma(\theta) \rho, \quad \xi^{k}=\xi^{k}(\theta, \rho),
$$


where $\gamma(\theta)$ is some arbitrary function of $\theta, \xi^{k}(\theta, \rho)$ has to satisfy

$$
\partial_{\rho} \xi^{k}=-\frac{1}{4} g_{(0)}^{k i} \partial_{i} \gamma(\theta)
$$

The action of this diffeomorphism on $\sigma(t, \theta, \rho), g_{i j}(t, \theta, \rho)$ and $A_{i}(t, \theta, \rho)$ is then given by

$$
\begin{gathered}
\mathcal{L}_{\xi} \sigma=\gamma(\theta) \rho \partial_{\rho} \sigma-2 \rho^{2} A_{k} g_{(0)}^{k i} \partial_{i} \gamma+\xi^{i} \partial_{i} \sigma \\
\mathcal{L}_{\xi} g_{i j}=\gamma(\theta)\left(\rho \partial_{\rho} g_{i j}-g_{i j}\right)+\frac{\rho^{2}}{t}\left(A_{i} \partial_{j} \gamma+A_{j} \partial_{i} \gamma\right)+\nabla_{i}^{(g)} \xi_{j}+\nabla_{j}^{(g)} \xi_{i} \\
\mathcal{L}_{\xi} A_{i}=\gamma(\theta)\left(\rho \partial_{\rho} A_{i}+A_{i}\right)+\frac{t}{4 \rho}\left(\partial_{i} \gamma-g_{k i} g_{(0)}^{k j} \partial_{j} \gamma\right)+\xi^{k} \partial_{k} A_{i}+\partial_{i} \xi^{k} A_{k}
\end{gathered}
$$

Notice that although there appears to be a term (the one proportional to $t$ ) in the right hand side of (6.6) which destroys its asymptotic behavior, this is not really true because $\partial_{i} \gamma-g_{k i} g_{(0)}^{k j} \partial_{j} \gamma$ vanishes as $1 / t$ in the limit where $t \rightarrow \infty$. One can see from (6.5) that the diffeomorphism generated by (6.2) acts on the metric $g_{i j}^{(0)}(\theta, \rho)$ in the same way as the diffeomorphism that was found in [28] to generate the asymptotic conformal structure of asymptotically Anti-de Sitter space. In particular, it acts on the metric $g_{i j}^{(0,0)}(\theta)$, the metric on the asymptotic boundary $\Sigma$ of the light-cone, as a conformal transformation

$$
\mathcal{L}_{\xi} g_{i j}^{(0,0)}=-\gamma(\theta) g_{i j}^{(0,0)}
$$

Thus, our analysis demonstrates that the conformal structure present on each AdS slice extends to the conformal structure associated with the boundary of the light-cone of $(d+2)$-dimensional asymptotically Minkowski space-time.

The analysis done so far was restricted to the region $\mathcal{A}_{-}$. A similar analysis can be done in the regions $\mathcal{A}_{+}$and $\mathcal{D}$. In fact the conformal structure near the boundary $\Sigma_{d}^{-}$of the past light-cone is analytic across the light-cone and extends from region $\mathcal{A}_{-}$to region $\mathcal{D}$. This can be seen by introducing new light-cone coordinates $u=-\frac{t}{\rho^{1 / 2}}, v=t \rho^{1 / 2}$ so that

$$
u v=-t^{2} \quad, \quad \frac{v}{u}=-\rho
$$

In terms of these coordinates the past light-cone is defined by $v=0$. The boundary $\Sigma_{d}^{-}$ of the light-cone is now at $u=-\infty$. In terms of the new coordinates the metric (6.1) takes the light-cone form (for simplicity we discard the subleading $1 / t$-terms in the metric (6.1))

$$
d s^{2}=d u d v+u^{2} g_{i j}^{(0)}(\theta, v / u) d \theta^{i} d \theta^{j}
$$

The boundary of the light-cone is at $u=-\infty$, and the $\rho$-expansion of the metric components now becomes an expansion in $v / u$. In the light-cone form (6.9) the metric is analytic across the past light-cone (at $v=0$ ) and extends from $\mathcal{A}_{-}$to $\mathcal{D}$ everywhere in the neighborhood of the boundary of the past-light cone. 
The diffeomorphism (6.2) now takes the form

$$
\xi^{v}=\frac{1}{2} v \gamma(\theta) \quad, \quad \xi^{u}=-\frac{1}{2} u \gamma(\theta), \quad \xi^{i}=\xi^{i}(u, v, \theta) .
$$

The form of the functions $\xi^{i}(\theta, u, v)$ is constrained by the equations

$$
\partial_{u} \xi^{i}=-\frac{v}{4 u^{2}} g_{(0)}^{i k}(\theta, v / u) \partial_{k} \gamma, \quad \partial_{v} \xi^{i}=\frac{1}{4 u} g_{(0)}^{i k}(\theta, v / u) \partial_{k} \gamma
$$

Since these are two equations there exists a consistency condition $\partial_{u} \partial_{v} \xi^{i}=\partial_{v} \partial_{u} \xi^{i}$. It reduces to a condition $\left(v \partial_{v}+u \partial_{u}\right) g_{i j}^{(0)}=0$ which does hold since the metric components in (6.9) are functions of $v / u$. One can see from (6.11) that $\xi^{i}$ is a function of the ratio $v / u$ of the light-cone coordinates. The first term $\xi_{(0)}^{i}$ in the expansion of $\xi^{i}(\theta, v / u)$ near the boundary of the light-cone is proportional to $v / u$ and solving (6.11) to this order we find that $\xi_{(0)}^{i}=\frac{v}{4 u} g_{(0,0)}^{i k} \partial_{k} \gamma(\theta)$.

The components $g_{i j}^{(0)}(\theta, v / u)$ transform under these diffeomorphisms according to

$$
\mathcal{L}_{\xi} g_{i j}^{(0)}=\gamma(\theta)\left(v \partial_{v} g_{i j}^{(0)}-u^{-1} \partial_{u}\left(u^{2} g_{i j}^{(0)}\right)\right)+\nabla_{i}^{(0)} \xi_{j}+\nabla_{j}^{(0)} \xi_{i} .
$$

Of course, equations (6.10), (6.11) and (6.12) are just the light-cone form of the previous equations (6.2), (6.3) and (6.5) that were written in terms of the coordinates $(t, \rho)$. However, the analyticity of the conformal structure across the light-cone is not obvious in those coordinates.

We conclude that there indeed exists a conformal symmetry associated with the boundary of the light-cone in the metric (6.1) or (6.9). This symmetry is generated by a certain bulk diffeomorphism (6.10), (6.11) and can be viewed as an extension of the conformal group existing on each EAdS slice to a symmetry of the bulk.

\subsection{BMS}

It should be noted that the group of all diffeomorphisms preserving the structure of the metric (6.1) is larger than just (6.2) and includes also $t$-dependent diffeomorphisms. The full analysis of diffeomorphisms of this type is given in Appendix C. We also find the generators of the well-known BMS group as a particular case of these $t$-dependent asymptotic transformations. The vector generating these diffeomorphisms is

$$
\begin{array}{r}
\xi^{t}=f(\theta) \rho^{1 / 2}, \\
\xi^{\rho}=-\frac{2}{t} f(\theta) \rho^{3 / 2}, \\
\xi^{i}=\frac{1}{t} \rho^{3 / 2} g_{(0,0)}^{i j}(\theta) \partial_{j} f(\theta) .
\end{array}
$$

The BMS group acts at a subleading order in the $1 / t$ expansion and leaves the metric

$g_{i j}^{(0,0)}(\theta)$ on $\Sigma_{d}$ unchanged. The BMS transformations are the asymptotic symmetries of the class of metrics which approach Minkowski metric near null infinity. Our asymptotic condition (6.1) is less restrictive and requires only that there is a light-cone structure near the surface $\Sigma_{d}$, which can be viewed as a single point in the null infinity. Therefore the group of asymptotic symmetries in our case is much larger than just BMS and in fact contains it as a subgroup. Of course, one may wonder whether the group of asymptotic symmetries and in particular the BMS group is actually a symmetry of the dual theory. To analyze this issue we now turn to a study of the various symmetries of the system. 


\subsection{Symmetries of the dual theory}

First we recall that a solution of the field equations of a massless scalar field admits on the de Sitter slices near $\tau=+\infty$ a decomposition given in (5.31)

$$
\lim _{\tau \rightarrow+\infty} \phi(r, \tau, \theta)=\frac{1}{2 \pi i} \int_{\frac{d}{2}-i \infty}^{\frac{d}{2}+i \infty} d \lambda N_{\lambda}^{-1} r^{-\lambda}\left(e^{-\lambda \tau}{ }_{\text {out }} \mathcal{O}_{\lambda}^{<}(\theta)+i^{\lambda-d} e^{-(d-\lambda) \tau}{ }_{\text {out }} \mathcal{O}_{\lambda}^{>}(\theta)\right) .
$$

We have been applying holography in the $\tau$-direction, resulting in the infinite set of operators out $\mathcal{O}_{\lambda}^{<}(\theta)$ and ${ }_{\text {out }} \mathcal{O}_{\lambda}^{>}(\theta)$. Instead of working with an infinite set of operators parametrized by the continuous parameter $\lambda$, we could also have decided replace the continuous parameter by an extra coordinate, so that the dual theory seems to live in $d+1$ dimensions. One way to introduce such an extra coordinate is to define

$$
\phi^{<}(\xi, \theta)=\frac{1}{2 \pi i} \int_{\frac{d}{2}-i \infty}^{\frac{d}{2}+i \infty} d \lambda N_{\lambda}^{-1} \xi^{-\lambda} \text { out } \mathcal{O}_{\lambda}^{<}(\theta)
$$

and similarly

$$
\phi^{>}(\xi, \theta)=\frac{1}{2 \pi i} \int_{\frac{d}{2}-i \infty}^{\frac{d}{2}+i \infty} d \lambda N_{\lambda}^{-1} \xi^{-\lambda} i^{\lambda-d}{ }_{\text {out }} \mathcal{O}_{\lambda}^{>}(\theta)
$$

In other words, the new coordinate $\xi$ arises by doing an inverse Mellin transform over the parameter $\lambda$. In constructing $\phi^{<,>}(\xi, \theta)$ we essentially did nothing but drop the leading exponential behavior in $\tau$ in (6.14). Thus $\phi^{<,>}(\xi, \theta)$ describe the boundary behavior of $\phi$ as $\tau \rightarrow \infty$ and in that sense it is similar to how one defines dual fields in AdS.

We have on purpose called the extra coordinate $\xi$. It clearly is very similar to $r$, but to make clear that we really want to view it as a new coordinate we gave it a new name. It is actually also closely related to $x_{+}$that appears in (2.8). Indeed, $r^{-\lambda} e^{-\lambda \tau}=\left(x_{+}\right)^{-\lambda}$, and therefore $\phi^{<}(\xi, \theta)$ describes the field $\phi$ as a function of $x^{+}$if we identify $x_{+}$with $\xi$. From this perspective it is also tempting to view the fields $\phi^{<,>}(\xi, \theta)$ as fields living on null infinity, and to view $\xi$ as the affine null coordinate of null infinity, so that the dual theory would actually live on null infinity. As we will see, this interpretation is somewhat problematic, but it would be interesting to study this further. On the other hand, $r^{-\lambda} e^{-(d-\lambda) \tau}=\left(x_{+}\right)^{-d / 2}\left(-x_{-}\right)^{d / 2-\lambda}$ and dropping the overall factor $\left(x_{+}\right)^{-d / 2}$ the operator $\phi^{>}(\xi, \theta)$ can be considered as function of $x_{-}$. Since $x_{-}$is the affine coordinate along the light-cone this suggests yet another interpretation of $\xi$ and of the dual theory.

We will now first study the action of the conformal group, or equivalently, the Lorentz group $S O(d+1,1)$. To write the explicit form of these generators in the de Sitter coordinates $(\tau, r, \theta)$ it is convenient to combine the angles $\theta$ and $r$ in a $d+1$ component vector with components $r_{i}$, so that $r^{2}=\sum_{i} r_{i}^{2}$, and so that the angles parametrize the unit sphere at $r=1$. With this convention, the rotation and boost generators of Minkowski space are

$$
\begin{aligned}
M_{i j} & =r_{i} \frac{\partial}{\partial r_{j}}-r_{j} \frac{\partial}{\partial r_{i}} \\
K_{i} & =\frac{r_{i}}{r} \frac{\partial}{\partial \tau}+\tanh \tau\left(r \frac{\partial}{\partial r_{i}}-\frac{r_{i}}{r} \sum_{k} r_{k} \frac{\partial}{\partial r_{k}}\right)
\end{aligned}
$$


In order to determine the action of these generators on $\phi^{<,>}(\xi, \theta)$, the strategy is to first find the exact solution of the free field equation with asymptotic behavior determined by $\phi^{<,>}(\xi, \theta)$, e.g. using a bulk-boundary propagator, to act with the bulk generators (6.17) on the exact solutions and to extract the new asymptotic behavior $\left(\phi^{\prime}\right)^{<,>}(\xi, \theta)$. Luckily for the generators (6.17) we can work with the asymptotic behavior only and we find that

$$
\begin{aligned}
M_{i j} & =\xi_{i} \frac{\partial}{\partial \xi_{j}}-\xi_{j} \frac{\partial}{\partial \xi_{i}} \\
K_{i}^{<} & =\frac{\xi_{i}}{\xi}\left(\xi \frac{\partial}{\partial \xi}\right)+\left(\xi \frac{\partial}{\partial \xi_{i}}-\frac{\xi_{i}}{\xi} \sum_{k} \xi_{k} \frac{\partial}{\partial \xi_{k}}\right) \\
K_{i}^{>} & =\frac{\xi_{i}}{\xi}\left(-d-\xi \frac{\partial}{\partial \xi}\right)+\left(\xi \frac{\partial}{\partial \xi_{i}}-\frac{\xi_{i}}{\xi} \sum_{k} \xi_{k} \frac{\partial}{\partial \xi_{k}}\right)
\end{aligned}
$$

where as we did above we combined the angles and $\xi$ into a $d+1$ component vector $\xi^{i}$ with $\xi^{2}=\sum \xi_{i}^{2}$, and $K_{i}^{<}$describes the action of the boost generators on $\phi^{<}(\xi, \theta)$, and similarly for $K_{i}^{>}$and $\phi^{>}(\xi, \theta)$.

The form of the generators (6.18) is consistent with the fact that the operators $\phi_{\lambda}(\theta)$ are conformal operators with conformal weights $\lambda$ or $d-\lambda$. It is interesting to observe that the generators (6.18) are simple differential operators when acting on $\phi^{<,>}(\xi, \theta)$. Therefore, they give rise to Ward identities for correlation functions in the dual theory. For example, a two point function of the form

$$
\left\langle\phi^{<}\left(\xi_{1}, \theta_{1}\right) \phi^{<}\left(\xi_{2}, \theta_{2}\right)\right\rangle
$$

should be annihilated by $K_{i, 1}^{<}+K_{i, 2}^{<}$. In conformal field theory the conformal Ward identities fix the form of a two-point function up to normalization, but here it only fixes it up to one unknown function. Indeed, one can show that the Ward identities imply that (6.19), up to contact terms, is of the form

$$
\left\langle\phi^{<}\left(\xi_{1}, \theta_{1}\right) \phi^{<}\left(\xi_{2}, \theta_{2}\right)\right\rangle=f_{<}\left(\xi_{1} \xi_{2}-\sum_{i} \xi_{1}^{i} \xi_{2}^{i}\right) \equiv f_{<}\left(\xi_{1} \xi_{2}\left(1-\cos \gamma\left(\theta_{1}, \theta_{2}\right)\right)\right)
$$

with some unknown function $f_{<}$. In terms of the operators $\phi_{\lambda}(\theta)$ the corresponding statement is that the two-point function is proportional to $\delta\left(\lambda_{1}-\lambda_{2}\right)$, but with a $\lambda$ dependent normalization, so that also in that formulation there is a function of one variable undetermined.

Similarly, one finds that (again up to contact terms)

$$
\left\langle\phi^{<}\left(\xi_{1}, \theta_{1}\right) \phi^{>}\left(\xi_{2}, \theta_{2}\right)\right\rangle=\xi_{2}^{-d} f\left(\xi_{1} \xi_{2}^{-1}\left(1-\cos \gamma\left(\theta_{1}, \theta_{2}\right)\right)\right)
$$

and

$$
\left\langle\phi^{>}\left(\xi_{1}, \theta_{1}\right) \phi^{>}\left(\xi_{2}, \theta_{2}\right)\right\rangle=\left(\xi_{1} \xi_{2}\right)^{-d} f_{>}\left(\xi_{1}^{-1} \xi_{2}^{-1}\left(1-\cos \gamma\left(\theta_{1}, \theta_{2}\right)\right)\right)
$$

To determine the form of the functions $f, f_{>}$and $f_{<}$one needs further input. A typical form of a two-point function is obtained by for example taking the first term proportional to $A(\lambda)$ in (5.15), and by dropping the $e^{-\lambda\left(\tau+\tau^{\prime}\right)}$ that appears there. The integral over $\lambda$ can be done explicitly, using the fact that the inverse Mellin transform of $\Gamma(a+z) \Gamma(b-z)$ is $\Gamma(a+b) x^{a}(1+x)^{-a-b}[29]$. This results

$$
\left\langle\mathcal{O}^{<}\left(\xi_{1}, \theta_{1}\right) \mathcal{O}^{>}\left(\xi_{2}, \theta_{2}\right)\right\rangle=\xi_{2}^{-d}\left(1+\frac{\xi_{1}}{\xi_{2}}\left(1-\cos \gamma\left(\theta_{1}, \theta_{2}\right)\right)\right)^{-d / 2}
$$


which is very similar to the asymptotic form of the Feynman propagator in coordinate space as $\tau, \tau^{\prime} \rightarrow \infty$. Similarly, we find that

$$
\left\langle\mathcal{O}^{>}\left(\xi_{1}, \theta_{1}\right) \mathcal{O}^{>}\left(\xi_{2}, \theta_{2}\right)\right\rangle=\left(\xi_{1} \xi_{2}\right)^{-d} \frac{\left(\xi_{1} \xi_{2}\right)^{d / 2}}{\left(1-\cos \gamma\left(\theta_{1}, \theta_{2}\right)\right)^{d / 2}} .
$$

Note, that calculating the correlation function (6.24) with the help of the inverse Mellin transform of product of two Gamma functions the result would contain the product of the delta function $\delta^{(d)}\left(\theta_{1}, \theta_{2}\right)$ and $\Gamma(0)$. This product, however, with the help of (A.2) can be recognized as the kernel $\left(1-\cos \gamma\left(\theta_{1}, \theta_{2}\right)\right)^{-d / 2}$.

The Lorentz or conformal generators (6.17) took a very simple form (6.18) when expressed in the new coordinate $\xi$. A crucial ingredient in the derivation of (6.18) was the fact that we were considering a massless scalar field. In general we would expect the conformal multiplet and the action of the conformal generators to depend on the field under consideration. For example, we can consider a massive scalar field with its decomposition given in terms of MacDonald functions in (3.11). Unfortunately, there does not seem to be a simple first order differential operator with eigenvalue $\nu$ when acting on $K_{\nu}(M \xi)$. Therefore, the massive generalization of (6.18) will be very complicated. The mode functions $\phi_{m}(x)$ in (3.11) are still solutions of the massive field equations on the AdS/dS slices, and the conformal group still acts in a simple way on these modes. We therefore believe that ultimately the description in terms of an infinite number of conformal fields is more useful than the description in terms of the additional coordinate $\xi$.

Besides Lorentz invariance, the bulk theory is also translationally invariant. Since our construction relies on a choice of light-cone, which is not invariant under translations, we may expect that translational invariance is not very manifest in our framework. Perhaps this is what is to be expected for a holographic dual description of Minkowski space, which is inherently non-local and employs one dimension less.

The explicit form of the translation generators, written in the same coordinates as used in (6.17), reads

$$
\begin{aligned}
P^{0} & =\frac{\cosh \tau}{r} \frac{\partial}{\partial \tau}-\frac{\sinh \tau}{r} \sum_{k} r_{k} \frac{\partial}{\partial r_{k}} \\
P^{i} & =-\frac{r_{i}}{r} \frac{\sinh \tau}{r} \frac{\partial}{\partial \tau}+\frac{1}{r \cosh \tau}\left(r \frac{\partial}{\partial r_{i}}\right)+\frac{r_{i}}{r^{2}} \frac{\sinh ^{2} \tau}{\cosh \tau} \sum_{k} r_{k} \frac{\partial}{\partial r_{k}} .
\end{aligned}
$$

Consider now the first term in (6.14), i.e. the one involving $\mathcal{O}^{<}(\theta)$. Acting with $P^{0}$ on this we obtain

$$
\frac{1}{2 \pi i} \int_{\frac{d}{2}-i \infty}^{\frac{d}{2}+i \infty} d \lambda N_{\lambda}^{-1}(-\lambda) r^{-\lambda-1} e^{-(\lambda+1) \tau}{ }_{\text {out }} \mathcal{O}_{\lambda}^{<}(\theta) .
$$

If we would drop the $e^{-(\lambda+1) \tau}$ exponentials, we would from this deduce that

$$
P^{0} \phi^{<}(\xi, \theta)=\frac{\partial}{\partial \xi} \phi^{<}(\xi, \theta) .
$$

However, this answer cannot be correct. For example, any two-point function of the form (6.20) cannot be annihilated by $P_{1}^{0}+P_{2}^{0}$ unless it vanishes identically. The mistake we 
made is to work only with the asymptotic form of the fields, neglecting subleading pieces that are necessarily there in the exact solution to the field equation. Subleading terms can mix with the result in (6.26), and this changes the action of $P^{0}$. We have not worked out the full detailed form of $P^{0}$ and $P^{i}$, but a useful perspective on these operators is obtained by looking at plane waves in the decomposition given in (3.7) and in particular in (5.28). Given a plane wave with momentum $k$, we denote the associated $\xi, \theta$-dependent fields by $W_{k}^{<,>}(\xi, \theta)$. One can work out the explicit form of these fields. One finds that $W_{k}^{>}(\xi, \theta)$ is some function of $k_{0} \xi$ times $\delta^{(d)}\left(\theta_{k}, \theta\right)$, where as before $\theta_{k}$ indicates the point on the $d$-sphere given by the $d+1$ dimensional unit vector $k_{i} / k_{0}$ (recall that we are still discussing massless scalar fields here). On the other hand, we have that

$$
W_{k}^{<}(\xi, \theta)=\exp \left(\frac{i}{2}\left(k_{0} \xi-\sum_{i} k_{i} \xi_{i}\right)\right)
$$

This shows that $P^{0}$ cannot be equal to $\partial / \partial \xi$. A plane wave has $P^{0}$ eigenvalue $i k_{0}$, whereas $\partial / \partial \xi$ acting on (6.28) has eigenvalue

$$
\frac{\partial}{\partial \xi} W_{k}^{<}(\xi, \theta)=\frac{i}{2 \xi}\left(k_{0} \xi-\sum_{i} k_{i} \xi_{i}\right) .
$$

There is no simple operator for which (6.28) has eigenvalue $i k_{0}$. Therefore, the easiest way to study translation invariance would be to rewrite the fields $\phi^{<}(\xi, \theta)$ in terms of momentum eigenstates such as (6.28), in terms of which translation invariance simply boils down to momentum conservation. Incidentally, the fields (6.28) are, up to a factor of two, simply the restriction of a plane wave to the light-cone. This suggest yet another interpretation for $\xi$, namely as a null coordinate along the light-cone. Since all AdS and $\mathrm{dS}$ slices asymptote to the light cone, this is perhaps the most natural interpretation of $\xi$.

A further input on the possible structure of the translations can be gained from the looking at the explicit form of the conformal operators (5.25) given in section 5.2. We find that

$$
\begin{aligned}
& \phi^{>}(\xi, \theta)=\int \frac{d \lambda}{2 \pi i} \xi^{-\lambda} \phi^{d-\lambda}(\theta) \\
& =\xi^{-d / 2} \int_{0}^{\infty} d k k^{d-1 / 2}\left(i^{-d / 2} e^{-i k \xi} a_{k}(k, \theta)+i^{d / 2} e^{i k \xi} a_{k}^{+}(k, \theta)\right)
\end{aligned}
$$

where in the second line we take explicitly the integral over $\lambda$. Under the time translation $X_{0} \rightarrow X_{0}+b$ the creation/annihilation operators (5.19) transform as $a\left(k, \theta_{k}\right) \rightarrow$ $e^{-i k b} a\left(k, \theta_{k}\right), a^{+}\left(k, \theta_{k}\right) \rightarrow e^{+i k b} a^{+}\left(k, \theta_{k}\right)$. This induces a transformation for the operators $\phi^{>}(\xi, \theta)$

$$
\phi^{>}(\xi, \theta) \rightarrow \phi^{>^{\prime}}(\xi, \theta)=(\xi+b)^{d / 2} \xi^{-d / 2} \phi^{>}(\xi+b, \theta)
$$

The two-point function of the operators (6.30) is given by (6.24) and is obviously invariant under (6.31). A similar analysis for the <-operators gives rise to the form

$\phi^{<}(\xi, \theta)=\int_{0}^{\infty} d k k^{d-1 / 2} \int d \mu\left(\theta_{k}\right)\left(e^{-i \frac{k \xi}{2}\left(1-\cos \gamma\left(\theta, \theta_{k}\right)\right)} a_{k}\left(k, \theta_{k}\right)+e^{i \frac{k \xi}{2}\left(1-\cos \gamma\left(\theta, \theta_{k}\right)\right)} a_{k}^{+}\left(k, \theta_{k}\right)\right)($

However the explicit way of how translations act on the operators (6.32) is not transparent. The problem of finding this transformation eventually boils down to the above mentioned 
problem of finding the operator for which the exponent (6.28) would have an eigenvalue $i k$.

Besides translations, there are infinitely many other operators in the BMS group which are probably also very complicated; naively they seem to act on fields $\phi^{<}(\xi, \theta)$ as $f(\theta) \partial_{\xi}$, but as the example of $P^{0}$ demonstrated, this is not quite correct. In addition the BMS generators are not exact symmetries of Minkowski space, and we therefore do not expect them to annihilate the vacuum state of the dual theory, and for the same reason we also do not expect them to give rise to new Ward identities. At best they provide a set of spectrum generating operators, but more work is needed to verify whether this is indeed the case or not. Actually, this applies to all asymptotic symmetries described in the beginning of this section, not just to the BMS operators.

\section{Gravitational holographic description}

\subsection{General remarks}

In section 4 of this paper we have seen how for a scalar field in Minkowski space a dual description emerges by first reducing the scalar field to a set of massive fields on a the constant curvature (de Sitter or anti-de Sitter) slices of space-time. In this section we will attempt to make a first step towards generalizing this duality to the gravitational field. The field equations in this case are the usual non-linear Einstein equations (with vanishing cosmological constant)

$$
\mathcal{R}_{\alpha \beta}=0
$$

which define a Ricci-flat space-time, which appears to be the appropriate generalization of flat Minkowski space. Equation (7.1) should be solved subject to suitable boundary conditions for the metric. We choose so-called normal coordinates in which the metric has the form

$$
d s^{2}=d r^{2}+r^{2} g_{i j}(x, r) d x^{i} d x^{j}
$$

Notice that for concreteness we consider the region analogous to the region $\mathcal{D}$ of Minkowski space, where the coordinate $r$ plays the role of a radial coordinate and the metric $g_{i j}(x, r)$ is assumed to have an asymptotic expansion in powers of $1 / r$. The coordinates $\left\{x^{i}\right\}, i=$ $1, . ., d+1$ are the coordinates on the slices, in the previous section we had $x^{i}=\{\tau, \theta\}$.

The bulk diffeomorphisms preserving the form (7.2) are generated by a vector field $\xi=\left(\xi^{r}, \xi^{i}\right)$ such that

$$
\begin{aligned}
& \xi^{r}=\alpha(x), \quad \xi^{i}=\xi^{i}(x, r) \\
& \partial_{r} \xi^{i}=-\frac{1}{r^{2}} g^{i j}(x, r) \partial_{j} \alpha(x) .
\end{aligned}
$$

The metric components change according to

$$
\mathcal{L}_{\xi} g_{i j}(x, r)=\alpha(x) r^{-2} \partial_{r}\left(r^{2} g_{i j}\right)+\nabla_{i}^{(g)} \xi_{j}+\nabla_{j}^{(g)} \xi_{i}
$$

The first term $g_{i j}^{(0)}(x)$ in the expansion of $g_{i j}(x, r)$ is the metric on the asymptotic space-like boundary of space-time. In analogy with the AdS/CFT correspondence one 
could try to keep the metric $g_{i j}^{(0)}(x)$ fixed and one could try to solve the bulk Einstein equations subject to this Dirichlet condition. However, in the present case when the bulk metric is determined by equation (17.1) the metric $g_{i j}^{(0)}(x)$ is not arbitrary but has to satisfy (see 30, 31], 32])

$$
R_{i j}\left[g^{(0)}\right]=d g_{i j}^{(0)}
$$

This means that the boundary at space-like infinity is described by an Einstein metric of constant positive curvature.

Provided that a metric $g_{i j}^{(0)}(x)$ satisfying (7.5) is fixed one can try to develop the $1 / r$ expansion of the metric (7.2) and determine the other terms in the expansion of $g_{i j}(x, r)$. To some extent this was analyzed in [20] and it was found that the relation between the coefficients in the expansion of $g_{i j}(x, r)$ is differential. This is different from what happens in asymptotically Anti-de Sitter space, where the relations are algebraic [27]. This makes it difficult to solve the recurrence relations and to determine the coefficients in the series provided the first coefficient $g_{i j}^{(0)}(x)$ is given.

However, the holographic point of view we are trying to develop in this paper is not based on fixing boundary data at spatial infinity. It is based on fixing an infinite set of data near the boundaries of the light-cone, and the holographic reduction takes place along the anti-de Sitter slices, and not along the coordinate $r$. Thus, what we should really try to is to take the metric in the form (6.1), and to study the field equations for this metric in this form. Ideally, there should exist some separation of variables that allows us to separate the $r$-dependence from the dependence on the other coordinates, just as we did for the massless scalar field. In view of the non-linearity of the Einstein equations it is not clear exactly how we should separate the $r$-dependence, but assuming we have achieved this the $r$-dependence will be gone and it will be replaced by a dependence on some spectral parameter $\lambda$. The boundary data will then involve quantities such as $\sigma^{\lambda, \pm}(\theta), g_{i j}^{\lambda, \pm}(\theta)$ and $A_{i}^{\lambda, \pm}(\theta)$. The holographic reconstruction of the metric now requires us to determine the $\rho$-dependence, and those equations are indeed algebraic. We leave a more detailed study of these issues to future work and limit ourselves here to the discussion of one example, namely the relatively simple case of $(2+1)$-dimensional Minkowski space.

\subsection{Example: (2+1)-dimensional Minkowski space-time}

In three space-time dimensions the Riemann tensor is algebraically related to the Ricci tensor. This property was used in [33] to find a general solution to the three-dimensional Einstein equations with a negative cosmological constant.

In the case at hand, the fact that the Ricci tensor vanishes implies that the Riemann tensor vanishes as well,

$$
\mathcal{R}_{\alpha \beta \mu \nu}=0
$$

i.e. space-time is locally flat which is of course a well-known fact in three dimensions. This is a great simplification in solving (7.1) and in fact allows us to find an exact solution in a way similar to the analysis given in 33 .

By introducing the variable $\gamma_{i j}(x, r)=r^{2} g_{i j}(x, r)$ the equation (17.6) for the metric (17.2) reduces to the following set of equations

$$
\gamma^{\prime \prime}=\frac{1}{2} \gamma^{\prime} \gamma^{-1} \gamma^{\prime}
$$




$$
\begin{gathered}
R_{l i k j}[\gamma]=\frac{1}{4} \gamma_{i j}^{\prime} \gamma_{l k}^{\prime}-\frac{1}{4} \gamma_{i k}^{\prime} \gamma_{l j}^{\prime} \\
\nabla_{k}^{(\gamma)} \gamma_{i j}^{\prime}-\nabla_{j}^{(\gamma)} \gamma_{i k}^{\prime}=0
\end{gathered}
$$

where we denote $\gamma^{\prime}=\partial_{r} \gamma$. Differentiating equation (7.7) once again with respect to $r$ we find that

$$
\gamma^{\prime \prime \prime}=0
$$

Hence $\gamma$ is a quadratic function of $r$, i.e.

$$
\gamma(x, r)=r^{2} g_{(0)}+r g_{(1)}+g_{(2)}
$$

Inserting this back into equation (7.7) we find that

$$
g_{(2)}=\frac{1}{4} g_{(1)} g_{(0)}^{-1} g_{(1)}
$$

so that (17.10) can be written as a total square

$$
\gamma(x, r)=r^{2}\left(1+\frac{1}{2 r} g_{(1)} g_{(0)}^{-1}\right) g_{(0)}\left(1+\frac{1}{2 r} g_{(0)}^{-1} g_{(1)}\right)
$$

Next, to solve equation (7.8) we use the following identity, valid in two dimensions, that relates the Riemann tensor to the Ricci scalar,

$$
R_{l i k j}[\gamma]=\frac{1}{2} R[\gamma]\left(\gamma_{k l} \gamma_{i j}-\gamma_{j l} \gamma_{k i}\right)
$$

Inserting (7.10) into eq.(7.8), expanding in powers of $1 / r$ and using the above identity we find in the leading order that

$$
R\left[g_{(0)}\right]=2,
$$

i.e. the space at infinity is two-dimensional de Sitter space. This is a particular manifestation of the general behavior (7.5) of the asymptotically flat metric. At the next order in $1 / r$ equation (7.8) reduces to a single equation**

$$
\nabla_{(0)}^{i} \nabla_{(0)}^{j} g_{(1) i j}-\nabla_{(0)}^{2} \operatorname{Tr} g_{(1)}=0
$$

On the other hand, equation (7.9) is trivially satisfied to the leading order and in the next order gives rise to the equation

$$
\nabla_{(0)}^{k} g_{(1) k i}=\partial_{i} \operatorname{Tr} g_{(1)}
$$

We see that (7.13) is a consequence of (7.14). The equations (7.12) and (7.14) are the only constraints to be imposed on the terms in the expansion (17.10).

Notice that equation (7.14) means that the tensor $g_{(1) i j}-g_{(0) i j} \operatorname{Tr} g_{(1)}$, like a stressenergy tensor, is covariantly conserved with respect to the metric $g_{(0) i j}$. In fact, according

\footnotetext{
**Hereafter we use $g_{(0)}$ to define the trace, e.g. $\operatorname{Tr} g_{(1)}=\operatorname{Tr}\left(g_{(0)}^{-1} g_{(1)}\right)$.
} 
to the prescription of Brown and York [34 the quasi-local stress-energy tensor of a spacetime is defined on the boundary (placed at a fixed value of $r$ which then should be taken to infinity) as a variation of the gravitational action with respect to the boundary metric and takes the form

$$
\begin{aligned}
T_{i j}^{\text {bound }}[\gamma] & =-\frac{1}{8 \pi G}\left(K_{i j}-K \gamma_{i j}\right) \\
& =-\frac{1}{8 \pi G}\left(-2 r g_{i j}+r^{2}\left(\partial_{r} g_{i j}-g_{i j} \operatorname{Tr}\left(g^{-1} \partial_{r} g\right)\right)\right.
\end{aligned}
$$

This expression is divergent when $r$ is taken to infinity. This can be thought of as a UV divergence in the theory on de Sitter space. It can be regularized by adding a local counterterm

$$
T_{i j}^{\mathrm{ct}}[\gamma]=\frac{1}{4 \pi G} \frac{1}{r} \gamma_{i j}
$$

The total stress tensor defined as the sum of the two contributions,

$$
\begin{aligned}
T_{i j}[\gamma] & =T_{i j}^{\text {bound }}+T_{i j}^{\mathrm{ct}} \\
& =-\frac{1}{8 \pi G}\left(g_{(1) i j}-g_{(0) i j} \operatorname{Tr} g_{(1)}\right)
\end{aligned}
$$

then has a finite limit when $r$ is taken to infinity and this limit reproduces the expression in the second line of (17.17). We see that up to a factor the stress tensor $T_{i j}$ is exactly the tensor which enters (7.14) and thus should be covariantly conserved with respect to the metric $g_{i j}^{(0)}$,

$$
\nabla_{(0)}^{j} T_{i j}=0
$$

In the remainder of the discussion we will use units in which $8 \pi G=1$.

Although empty $(2+1)$-space-time is locally flat it becomes curved in the presence of matter. For instance, a point particle creates $\delta$-like conical singularity distributed along the particle world line. The stress-energy tensor (17.17) then determines the energy and angular momentum of the matter as measured at space-like infinity. In terms of $T_{i j}$ we have that

$$
g_{i j}^{(1)}=-T_{i j}+g_{i j}^{(0)} \operatorname{Tr} T .
$$

Taking into account (17.10) and (7.11) we see that the boundary stress tensor $T_{i j}(x)$ and the boundary metric $g_{i j}^{(0)}(x)$ subject to constraint (7.12) completely determine the bulk metric satisfying the Ricci-flat equation (7.1) in three dimensions. This is similar to what we had in the case of negative cosmological constant in three dimensions [33]. The difference is that in the latter case the trace of the boundary stress tensor is fixed and no constraint on the boundary metric arises. The stress tensor on the de Sitter boundary of asymptotically Minkowski space-time should be covariantly conserved with respect to the metric $g_{i j}^{(0)}$ but is otherwise arbitrary. In particular, there is no restriction on the trace of $T_{i j}$. This would be quite natural if we were doing a holographic reduction in the radial direction, since then $T_{i j}$ should represent a stress tensor of a boundary theory which is holographically dual to a theory in the bulk. But we emphasize once more that this not the type of holographic reduction we are attempting to do in this paper. 
To the leading order the $r$-dependent diffeomorphism (17.3) is generated by the vector field

$$
\xi^{r}=\alpha(x), \quad \xi^{i}=\frac{1}{r} g_{(0)}^{i j} \partial_{j} \alpha(x)
$$

where $\alpha(x)$ is arbitrary function on the de Sitter boundary. The boundary metric $g_{i j}^{(0)}$ remains unchanged under this transformation while $g_{i j}^{(1)}$ transforms as

$$
\mathcal{L}_{\xi} g_{i j}^{(1)}=\alpha(x) g_{i j}^{(0)}+\nabla_{i}^{(0)} \nabla_{j}^{(0)} \alpha
$$

This transformation acts as

$$
\delta_{\alpha} T_{i j}=-\nabla_{i}^{(0)} \nabla_{j}^{(0)} \alpha+g_{i j}^{(0)} \nabla_{(0)}^{2} \alpha+\alpha g_{i j}^{(0)}
$$

on the stress tensor $T_{i j}$. It is easy to check that the r.h.s. in (7.22) is covariantly conserved on a two-dimensional space-time of constant curvature (7.12). It can be obtained by varying the following "dilaton-gravity" boundary action

$$
W_{\alpha}=\int d^{2} x \sqrt{g_{(0)}} \alpha(x)\left(R\left[g_{(0)}\right]-2\right)
$$

with respect to the metric. Note also that the variation of (17.23) with respect to $\alpha(x)$ gives rise to the equation (7.12).

Let us now show that on de Sitter space the stress tensor $T_{i j}$ can be determined by solving (7.18) provided its trace is some known function

$$
\operatorname{Tr} T=C(x) .
$$

We choose the metric

$$
d s^{2}=\frac{1}{z^{2}}\left(-d z^{2}+d \theta^{2}\right)=\frac{4}{\left(x_{+}-x_{-}\right)^{2}} d x_{+} d x_{-},
$$

where $x_{+}=\theta+z, x_{-}=\theta-z$, to describe the two-dimensional de Sitter space. This coordinate system covers half of de Sitter space, the boundary then being at $z=0$. The trace equation (7.24) then determines the (+-) component of the stress tensor

$$
T_{+-}=\frac{1}{\left(x_{+}-x_{-}\right)^{2}} C\left(x_{+}, x_{-}\right) .
$$

From (17.18) we obtain a couple of differential equations

$$
\partial_{-} T_{++}=-\frac{1}{\left(x_{+}-x_{-}\right)^{2}} \partial_{+} C, \quad \partial_{+} T_{--}=-\frac{1}{\left(x_{+}-x_{-}\right)^{2}} \partial_{+} C .
$$

A solution can be easily found and it takes the form

$$
\begin{gathered}
T_{++}\left(x_{+}, x_{-}\right)=f\left(x_{+}\right)-\int^{x_{-}} \frac{d v}{\left(x_{+}-v\right)^{2}} \partial_{+} C\left(x_{+}, v\right) \\
T_{--}\left(x_{+}, x_{-}\right)=h\left(x_{-}\right)-\int^{x_{+}} \frac{d u}{\left(x_{-}-u\right)^{2}} \partial_{-} C\left(u, x_{-}\right),
\end{gathered}
$$


where $f\left(x_{-}\right)$and $h\left(x_{+}\right)$are arbitrary functions of $x_{-}$and $x_{+}$respectively. The boundary stress tensor (7.26), (7.28) and eventually the bulk metric are determined by specifying the functions $f\left(x_{+}\right), h\left(x_{-}\right)$and $C\left(x_{+}, x_{-}\right)$. Let us now return to the holographic interpretation of this solution. The central idea proposed in this paper is to reduce everything to holographic data on the boundary of the light-cone, which in the present case is a circle located at $z=0$ and parametrized by the angle coordinate $\theta$. Thus, from the point of view of the holographic reconstruction it is important to see what the minimal data is that we need to fix on this circle in order to reconstruct the full $(2+1)$-dimensional spacetime. We see that the values of the functions $f\left(x_{+}\right)$and $h\left(x_{-}\right)$at late times $z$ are completely determined by their values at $z=0$, i.e. $f(\theta)$ and $h(\theta)$. On the other hand, the function $C\left(x_{+}, x_{-}\right)$seems to require an infinite set of functions to be specified at $z=0$, namely all coefficients in the expansion

$$
C\left(x_{+}, x_{-}\right)=\sum_{n=0}^{\infty} C_{n}(\theta) z^{n},
$$

where $\theta=\frac{1}{2}\left(x_{+}+x_{-}\right)$and $z=\frac{1}{2}\left(x_{+}-x_{-}\right)$. However, most of this infinite set of functions can be gauged away by the coordinate transformation (7.20). As is seen from (7.22) this transformation parametrised by

$$
\alpha\left(x_{+}, x_{-}\right)=\sum_{n=0}^{\infty} \alpha_{n}(\theta) z^{n}
$$

transforms the function $C\left(x_{+}, x_{-}\right)$. Specifically, the coefficients in the expansion (7.29) transform as follows

$$
\delta_{\alpha} C_{n}(\theta)=\frac{1}{4} \alpha_{n-2}^{\prime \prime}(\theta)-(n-2)(n+1) \alpha_{n}(\theta) .
$$

We see that the parameter function $\alpha\left(x_{+}, x_{-}\right)$contains enough freedom to eliminate almost all functions $C_{n}(\theta)$ with one exception: the same parameter $\alpha_{0}(\theta)$ is used to transform both $C_{0}(\theta)$ and $C_{2}(\theta)$ so that one can not eliminate both. In fact the combination

$$
c(\theta)=C_{2}(\theta)-2 C_{0}^{\prime \prime}(\theta)
$$

is invariant under the transformation, $\delta_{\alpha} c(\theta)=0$. Thus, the functions $c(\theta)$ together with $f(\theta)$ and $h(\theta)$ form the only independent and "gauge-invariant" set of data which should be specified at the boundary of the light-cone. These data are sufficient to holographically reconstruct the whole three-dimensional metric. Actually, this is a quite subtle statement. Asymptotic diffeomorphisms do not in general extend to smooth diffeomorphisms of the entire space, and acting with them typically generates singularities. The diffeomorphisms used to reduce the infinite set of functions $C_{n}(\theta)$ to just one function of $\theta$ are presumably of this form. Therefore, if we are interested in smooth solutions, it is probably better to work with the infinite set $C_{n}(\theta)$ instead. For this infinite set the existence of a globally smooth solution is obviously also not guaranteed, and more work is required to determine which sets of $C_{n}(\theta)$ correspond to smooth solutions.

We finish this section with a comment on a RG interpretation of the $(2+1)$-dimensional metric. As we have seen above, if we view $r$ as the direction in which we do the holographic reduction the standard interpretation of the radial position $r$ of the boundary is as a UV 
cut-off in the boundary theory. The dependence of the metric on $r$ can be viewed as some kind of RG-flow. In this respect it is interesting to see whether this flow is irreversible so that some kind of analog of the C-theorem could be found. Remarkably the flow, as it is dictated by the bulk Einstein equation (17.1), is indeed irreversible. In order to see this, we consider the quasi-local stress tensor (17.15) and examine how its trace computed with respect to the induced metric $\gamma_{i j}$ changes as a function of $r$. Using equation (7.7) we find that

$$
\partial_{r} \operatorname{Tr} T^{\text {bound }}=-\frac{1}{2} \operatorname{Tr}\left(\gamma^{-1} \gamma^{\prime} \gamma^{-1} \gamma^{\prime}\right)<0
$$

However, it is not clear whether the bulk gravitational dynamics drives the flow to a fixed point which would correspond to a conformal field theory on de Sitter boundary of Minkowski space. In any case this is not the point of view we want to pursue further.

\section{Conclusion}

In this paper we have proposed a holographic reduction of Minkowski space. The central idea was to perform a holographic reduction along the Euclidean anti-de Sitter and de Sitter slices in which Minkowski space is naturally foliated. The end product is that we obtained an infinite set of conformal operators on the two spheres that form the conformal boundary of the light-cone. For a massless scalar field in the bulk we gave a precise definition of a Minkoswki/CFT duality which also extends to interacting fields. We have mainly considered cases where we took all points either inside Euclidean anti-de Sitter space, or inside de Sitter space, but the results in this paper can be extended to the mixed case as well.

It is perhaps worth pausing for a moment to see what we have learnt about de Sitter space holography from this approach. The first lesson seems to be that the natural conformal weights to emerge on de Sitter space are complex ones. Since a massless scalar field in Minkowski space describes a unitary theory, and carries a unitary representation of the Lorentz group, the corresponding theory on de Sitter space and its holographic dual should also be unitary. All this is evidence in favor of the picture of the dual of de Sitter space advocated in [17]. There, it was proposed that the dual CFT has non-standard hermiticity conditions, such that complex conformal weights do not violate unitarity, and $S O(d+1,1)$ is unitarily realized. In fact, there are two CFT's, one living on each boundary, and full correlation functions are given by evaluating them in the product theory in a suitable entangled state. This entangled state is the analogue of the S-matrix, and the set of correlation functions on each of the spheres at infinity represents the in and out Hilbert spaces.

Clearly, it should also be possible to study other manifolds that can be sliced in (anti)de Sitter slices, and to study holography in those cases. It would also be interesting to

study e.g. anti-de Sitter space in the Poincare patch, to view this as a slicing with Minkowski slices and to see if the known holography for anti-de Sitter space is consistent with our proposed Minkowski space holography.

A further extension and a crucial test of our idea would be to study the Schwarzschild metric, and to see how the entropy of the Schwarzschild black hole is reflected in the dual CFT. For this, we should first further develop the extension of our method to the gravitational field, for which only a few first steps were made in sections 6,7. Especially, it 
is not yet clear exactly which data at infinity we should specify in order to reconstruct the bulk geometry, and whether this data allows for an interpretation in terms of an infinite set of stress-energy tensors.

One can imagine many other extensions. We have only briefly considered massive scalar fields, and it would be desirably to understand these better. Another thing that comes to mind is a supersymmetric generalization (or a generalization to string theory). Since supersymmetries close into translation generators and we already saw that translation symmetry does not take on a very simply form, we suspect that it will also be difficult to keep supersymmetry manifest in the formulation. The fact that translation invariance is implemented in such a cumbersome way is perhaps to be expected for a holographic dual description of Minkowski space, but it would be nice to have a clearer understanding of this point.

Even though free massive particles will not approach the boundary of the light-cone, but rather go to timelike infinity, we still saw that it is possible to describe them in terms of data on the boundary of the light cone. The situation gets even more confusing for strongly interacting degrees of freedom, such as those that appear in confining gauge theories, and for which the LSZ formalism does not seem appropriate. We nevertheless still expect that the physics of such degrees of freedom will be captured by suitable data on the two spheres that form the boundary of the light-cone.

Of course, we are not living in Minkowski space, and it is an even more urgent question to find a holographic dual description of a realistic time-dependent cosmological solution. Nevertheless, Minkowski space is still a good approximation for many physical questions, and understanding Minkowski space is definitely a step in the right direction. Incidentally, four-dimensional Minkowski space reduces in this philosophy to a theory on two twospheres, which have an infinite dimensional conformal symmetry, and this may lead to an interesting role of the Virasoro algebra for four-dimensional physics.

ACKNOWLEDGEMENTS We would like to thank V. Balasubramanian, C. Hull, N. Lambert, D. Minic, H. Reall, K. Skenderis, M. Taylor-Robinson and E. Verlinde for discussions. S.S. would like to thank ITFA for hospitality during final stage of this work. Research of S.S. is supported by the grant DFG-SPP 1096, Stringtheorie. 


\section{Appendix}

\section{A Kernels and delta-functions}

The kernel

$$
\left(\cosh y-\sinh y \cos \gamma\left(\theta, \theta^{\prime}\right)\right)^{-\lambda}
$$

produces a delta-function concentrated at $\theta=\theta^{\prime}$ when $y \rightarrow \infty$. In order to see this and get the precise factor we take $\theta \simeq \theta^{\prime}$ and approximate $\cos \gamma\left(\theta, \theta^{\prime}\right) \simeq 1-\gamma^{2} / 2$. In the limit of large $y$ the kernel takes the form

$$
\frac{2^{2 \lambda} e^{-\lambda y}}{\left(\gamma^{2}+4 e^{-2 y}\right)^{\lambda}}
$$

By introducing the small parameter $\delta=2 e^{-y}$ this can be brought in the form

$$
2^{d} e^{-(d-\lambda) y}\left(\frac{\delta^{2 \lambda-d}}{\left(\gamma^{2}\left(\theta, \theta^{\prime}\right)+\delta^{2}\right)^{\lambda}}\right)
$$

where the term in the parenthesis generates the delta-function $\delta^{(d)}\left(\theta, \theta^{\prime}\right)$ on d-sphere up to some normalization constant. In order to determine this constant we consider the integral

$$
\lim _{\delta \rightarrow 0} \int_{S_{d}} \frac{\delta^{2 \lambda-d}}{\left(\gamma^{2}\left(\theta, \theta^{\prime}\right)+\delta^{2}\right)^{\lambda}}=\frac{2 \pi^{\frac{d}{2}}}{\Gamma\left(\frac{d}{2}\right)} \int_{0}^{\infty} \frac{d x x^{d-1}}{\left(x^{2}+1\right)^{\lambda}}
$$

where the first factor is the area of a (d-1)-sphere and in the integration over the azimuthal angle we changed the variable $\gamma$ to $\delta x$. The $x$-integration results in

$$
\int_{0}^{\infty} \frac{d x x^{d-1}}{\left(x^{2}+1\right)^{\lambda}}=\frac{1}{2} \frac{\Gamma\left(\frac{d}{2}\right) \Gamma\left(\lambda-\frac{d}{2}\right)}{\Gamma(\lambda)} .
$$

Putting things together we find that

$$
\lim _{y \rightarrow \infty}\left(\cosh y-\sinh y \cos \gamma\left(\theta, \theta^{\prime}\right)\right)^{-\lambda}=(2 \pi)^{\frac{d}{2}} 2^{\frac{d}{2}} \frac{\Gamma\left(\lambda-\frac{d}{2}\right)}{\Gamma(\lambda)} e^{-(d-\lambda) y} \delta^{(d)}\left(\theta, \theta^{\prime}\right) .
$$

A similar analysis can be done for the kernels

$$
\left(\sinh \tau-\cosh \tau \cos \gamma\left(\theta, \theta^{\prime}\right)+i \epsilon\right)^{-\lambda}
$$

in the limits $\tau \rightarrow+\infty$ or $\tau \rightarrow-\infty$. The integration over the azimuthal angle in this case is

$$
\int_{0}^{\infty} \frac{d x x^{d-1}}{\left(x^{2}-1+i \epsilon\right)^{\lambda}}=\int_{0}^{+i \infty} \frac{d z z^{d-1}}{\left(z^{2}-1\right)^{\lambda}}=i^{d-2 \lambda} \int_{0}^{\infty} \frac{d y y^{d-1}}{\left(y^{2}+1\right)^{\lambda}},
$$

where we first deformed the integration contour to the positive imaginary axis (the possible integral over the quarter-circle at infinity vanishes provided $\operatorname{Re}(2 \lambda-d)$ is slightly positive) and then changed variables $z \rightarrow i y$ that brings us back to the already known integral given above. Thus, we find that

$$
\begin{aligned}
& \lim _{\tau \rightarrow+\infty}\left(\sinh \tau-\cosh \tau \cos \gamma\left(\theta, \theta^{\prime}\right)+i \epsilon\right)^{-\lambda} \\
& =(2 \pi)^{d / 2} 2^{\frac{d}{2}} i^{(d-2 \lambda)} \frac{\Gamma\left(\lambda-\frac{d}{2}\right)}{\Gamma(\lambda)} e^{-(d-\lambda) \tau} \delta^{(d)}\left(\theta, \theta^{\prime}\right) .
\end{aligned}
$$


When $\tau \rightarrow-\infty$ the kernel diverges at $\gamma\left(\theta, \theta^{\prime}\right)=\pi$ and hence delta-function that is obtained in this limit

$$
\begin{aligned}
& \lim _{\tau \rightarrow-\infty}\left(\sinh \tau-\cosh \tau \cos \gamma\left(\theta, \theta^{\prime}\right) \pm i \epsilon\right)^{-\lambda} \\
& =(2 \pi)^{d / 2} 2^{\frac{d}{2}}(\mp i)^{d} \frac{\Gamma\left(\lambda-\frac{d}{2}\right)}{\Gamma(\lambda)} e^{(d-\lambda) \tau} \delta^{(d)}\left(\theta, \pi-\theta^{\prime}\right)
\end{aligned}
$$

is concentrated at the anti-podal points on the d-sphere.

\section{B Plane wave normalization}

The plane waves

$$
f_{k}\left(X_{0}, \mathbf{X}\right)=\frac{1}{\left[(2 \pi)^{d+1} 2 k\right]^{1 / 2}} e^{-i\left(k X_{0}-\mathbf{k X}\right)}
$$

are delta-function normalized

$$
\left(f_{k}, f_{p}\right)=\delta^{(d+1)}(\mathbf{k}, \mathbf{p})=k^{-d} \delta(k-p) \delta^{(d)}\left(\theta_{k}, \theta_{p}\right)
$$

with respect to the standard Klein-Gordon scalar product which is defined as

$$
\left(f_{k}, f_{p}\right)=i \int_{\Sigma} d \sigma\left(f_{k}^{*} \partial_{n} f_{p}-\partial_{n} f_{k}^{*} f_{p}\right)
$$

provided the hypersurface $\Sigma$ (with the normal vector $n$ ) is a surface of constant Minkowski time $X_{0}$.

However, for our purposes we would like to choose a hypersurface associated with the boundary of the light-cone. More specifically we define $\Sigma_{\text {out }}$ as a hypersurface consisting of two components: a surface $\tau=+\infty$ in the region $\mathcal{D}$ and a surface $y=+\infty(t>0)$ in the region $\mathcal{A}$. The hypersurface $\Sigma_{\text {out }}$ thus surrounds the boundary $S_{d}^{+}$of the light-cone. Similarly we define $\Sigma_{\text {in }}=\{\mathcal{D}: \tau=-\infty\} \cup\{\mathcal{A}: y=+\infty(t<0)\}$ near the boundary $S_{d}^{-}$.

Here we show that same normalization (B.2) still holds if we choose $\Sigma_{\text {out }}$ (or $\Sigma_{\text {in }}$ ) in the scalar product (B.3). We demonstrate this explicitly for the surface $\Sigma_{\text {out }}$. The nonvanishing components of the normal vector $n$ are $n^{\tau}=r^{-1}$ (in region $\mathcal{D}$ ) and $n^{y}=t^{-1}$ (in region $\mathcal{A}$ ). Hence each component gives

$$
\begin{aligned}
& \int_{\Sigma_{\text {out }}} d \sigma \partial_{n}=\int_{\tau \rightarrow+\infty}(\cosh \tau)^{d} d \mu(\theta) \int_{0}^{\infty} d r r^{d-1} \partial_{\tau} \\
& -\int_{y \rightarrow+\infty}(\sinh y)^{d} d \mu(\theta) \int_{0}^{\infty} d t t^{d-1} \partial_{y}
\end{aligned}
$$

in the scalar product (B.3). As $y \rightarrow+\infty$ the plane wave asymptotically behaves as

$$
e^{ \pm i\left(k X_{0}-\mathbf{k X}\right)}=\frac{2^{d} \pi^{\frac{d}{2}}}{2 \pi i} \int d \lambda( \pm i)^{\lambda} \Gamma\left(\lambda-\frac{d}{2}\right) k^{-\lambda} t^{-\lambda} e^{-(d-\lambda) y} \delta^{(d)}\left(\theta, \theta_{k}\right)
$$

where we again assumed that $\operatorname{Re}\left(\lambda-\frac{d}{2}\right)$ is slightly positive which allows us to drop the term proportional to $e^{-\lambda y}$. Similarly in region $\mathcal{D}$ the plane wave asymptotically behaves for $\tau \rightarrow+\infty$ as

$$
e^{ \pm i\left(k X_{0}-\mathbf{k X}\right)}=\frac{2^{d} \pi^{\frac{d}{2}}}{2 \pi i} \int d \lambda( \pm i)^{d-\lambda} \Gamma\left(\lambda-\frac{d}{2}\right) k^{-\lambda} r^{-\lambda} e^{-(d-\lambda) \tau} \delta^{(d)}\left(\theta, \theta_{k}\right)
$$


Inserting these into the definition of the scalar product (B.3)-(B.4 and taking into account that

$$
\int_{0}^{\infty} d r r^{d-\lambda-\lambda^{\prime}-1}=2 \pi i \delta\left(\lambda+\lambda^{\prime}-d\right)
$$

we find

$$
\begin{aligned}
& \left(f_{k}, f_{p}\right)_{\Sigma_{\mathrm{out}}}=-\frac{(2 \pi)^{-1}}{\sqrt{k p}} p^{-d} \delta^{(d)}\left(\theta_{k}, \theta_{p}\right) \\
& \frac{1}{2 \pi i} \int d \lambda \sin \frac{\pi}{2}(d-2 \lambda)(d-2 \lambda) \Gamma\left(\lambda-\frac{d}{2}\right) \Gamma\left(\frac{d}{2}-\lambda\right)\left(\frac{k}{p}\right)^{-\lambda}
\end{aligned}
$$

for the scalar product over $\Sigma_{\text {out }}$. Notice that the powers of $i$ coming from the contributions of each component nicely combine into $i^{d-2 \lambda}-i^{2 \lambda-d}=2 i \sin \frac{\pi}{2}(d-2 \lambda)$. Using that the Gamma function satisfies

$$
\Gamma\left(\lambda-\frac{d}{2}\right) \Gamma\left(\frac{d}{2}-\lambda\right)=-\frac{2 \pi}{(d-2 \lambda) \sin \frac{\pi}{2}(d-2 \lambda)}
$$

the $\lambda$-integration in (B.7) reduces to a simple integral

$$
\frac{1}{2 \pi i} \int_{\frac{d}{2}-i \infty}^{\frac{d}{2}+i \infty} d \lambda\left(\frac{k}{p}\right)^{-\lambda}=k \delta(k-p) .
$$

Thus, equation (B.7) is indeed identical to (B.2).

Note that this result could have been anticipated. Indeed, suppose that we add to the hypersurface $\Sigma_{\text {out }}$ the surface $t=+\infty$ in the region $\mathcal{A}$. The resulting hypersurface can be thought of as a deformation of the surface $X_{0}=+\infty$ for which the standard normalization (B.2) holds. Now, the Klein-Gordon scalar product (B.3) should not change under any deformation of the surface $\Sigma$. On the other hand, the contribution of the surface $t=+\infty$ to the scalar product vanishes provided $\operatorname{Re}(2 \lambda-d)$ is small but positive. Hence, the whole contribution to the scalar product should come entirely from the hypersurface $\Sigma_{\text {out }}$. We however found it useful to give an explicit calculation of the scalar product over $\Sigma_{\text {out }}$. This serves both as an illustration of our techniques as well as a consistency check.

\section{Diffeomorphisms}

Here we perform analysis of the diffeomorphisms preserving the asymptotic structure of the metric (6.1). Suppose that the diffeomorphism is generated by a vector field $\xi=$ $\left(\xi^{t}, \xi^{\rho}, \xi^{i}\right)$. By requiring that $\mathcal{L}_{\xi} G_{t t}=0$ we obtain $\partial_{t} \xi^{t}=0$ from which we find that the $t$-component of the vector $\xi$ does not depend on $t$ so that

$$
\xi^{t}=\alpha(\theta, \rho)
$$

where $\alpha(\theta, \rho)$ is arbitrary function of $\theta$ and $\rho$.

The condition $\mathcal{L}_{\xi} G_{t \rho}=0$ leads to

$$
\partial_{t} \xi^{\rho}=\frac{4 \rho^{2}}{\left(1+\frac{1}{t} \sigma\right)}\left(t^{-2} \partial_{\rho} \alpha-t^{-1} A_{i} \partial_{t} \xi^{i}\right) .
$$


and from the condition $\mathcal{L}_{\xi} G_{t i}=0$ we find that

$$
\partial_{t} \xi^{i}=\rho\left(t^{-2} g^{j i} \partial_{i} \alpha-t^{-1} g^{j i} A_{i} \partial_{t} \xi^{\rho}\right)
$$

Multiplying equation (C.3) by $A_{j}$ and inserting the result into equation (C.2) we find the equations for the $t$-derivatives of $\xi^{\rho}$ and $\xi^{i}$ :

$$
\begin{gathered}
\partial_{t} \xi^{\rho}=\frac{1}{\left(1+\frac{1}{t} \sigma-\frac{4 \rho^{3}}{t^{2}} A^{2}\right)} \frac{4 \rho^{2}}{t^{2}}\left(\partial_{\rho} \alpha-\frac{\rho}{t} A^{i} \partial_{i} \alpha\right) \\
\partial_{t} \xi^{i}=\frac{\rho}{t^{2}} g^{j i} \partial_{i} \alpha-\frac{4 \rho^{3}}{t^{3}} \frac{g^{j i} A_{i}}{\left(1+\frac{1}{t} \sigma-\frac{4 \rho^{3}}{t^{2}} A^{2}\right)}\left(\partial_{\rho} \alpha-\frac{\rho}{t} A^{i} \partial_{i} \alpha\right),
\end{gathered}
$$

where we introduced $A^{2}=A_{i} g^{i j} A_{j}$ and all $i$-indexes are raised with help of the metric $g_{i j}(t, \theta, \rho)$.

Now, let us make a substitution

$$
\begin{aligned}
\xi^{\rho} & =\gamma(\theta, \rho)+\frac{1}{t} \beta(t, \theta, \rho) \\
\xi^{i} & =\xi_{(0)}^{i}(\theta, \rho)+\frac{1}{t} \xi_{(1)}^{i}(\theta, \rho)+. .
\end{aligned}
$$

The $t^{2}$ term in the $G_{\rho \rho}$ component of the metric is fixed and should not be changed under the diffeomorphism. This implies $\rho \partial_{\rho} \gamma(\theta, \rho)-\gamma(\theta, \rho)=0$ which in turn leads to

$$
\gamma(\theta, \rho)=\gamma(\theta) \rho
$$

where $\gamma(\theta)$ is an arbitrary function. Similarly, the $t^{2}$ term in $G_{i \rho}$ of the metric (6.1) vanishes. In order to preserve this condition the $t$-independent part of $\xi^{i}$ should satisfy

$$
\partial \rho \xi_{(0)}^{j}=-\frac{1}{4} g_{(0)}^{j i} \partial_{j} \gamma(\theta)
$$

The diffeomorphism generated by the vector field

$$
\xi=\left(0, \gamma(\theta) \rho, \xi_{(0)}^{j}(\theta, \rho)\right)
$$

is the only $t$-independent diffeomorphism that preserves the form (6.1).

As for the $t$-dependent parts of the functions, $\alpha(\theta, \rho)$ plays the role of the parameters of the transformation. Equations (C.4) and (C.5) determine $\beta(t, \theta, \rho)$ and $\xi_{(k>0)}^{i}(\theta, \rho)$ in terms of $\alpha(\theta, \rho)$. For the first terms in the $t$-expansion of these functions we find that

$$
\begin{aligned}
& \beta_{(0)}(\theta, \rho)=-4 \rho^{2} \partial_{\rho} \alpha(\theta, \rho), \\
& \beta_{(1)}=2 \rho^{2}\left(\sigma_{(0)} \partial_{\rho} \alpha+\rho A_{(0)}^{i} \partial_{i} \alpha\right)
\end{aligned}
$$

and

$$
\xi_{(1)}^{i}=\rho g_{(0)}^{i j} \partial_{j} \alpha(\theta, \rho)
$$

The subleading terms in $G_{\rho \rho}$ and $G_{i \rho}$ change under the diffeomorphisms and will give us the transformation law for the functions $\sigma(t, \theta, \rho)$ and $A_{i}(t, \theta, \rho)$ but we will not give 
the explicit form here. Also, by acting on the $G_{i j}$ part of the metric the diffeomorphism provides the transformation law for $g_{i j}(t, \theta, \rho)$, namely

$$
\begin{aligned}
& \mathcal{L}_{\xi} g_{i j}=\alpha(\theta, \rho)\left(\partial_{t}+\frac{2}{t} g_{i j}\right)+\xi^{\rho}\left(\partial_{\rho} g_{i j}-\frac{1}{\rho} g_{i j}\right) \\
& +\frac{\rho}{t}\left(A_{i} \partial_{j} \xi^{\rho}+A_{j} \partial_{i} \xi^{\rho}\right)+\nabla_{i}^{(g)} \xi_{j}+\nabla_{j}^{(g)} \xi_{i}
\end{aligned}
$$

where $\xi^{\rho}$ takes the form (C.6). The transformations generated by the $t$-independent part of the diffeomorphisms are given in (6.4), (6.6) and (6.5) in the main text.

It is interesting to note that the BMS transformation are a particular case of the $t$ dependent diffeomorphisms. Specifically, they are determined by the function $\alpha(\theta, \rho)=$ $f(\theta) \rho^{1 / 2}$ where $f(\theta)$ is an arbitrary function of the angle coordinate on the boundary $\Sigma$ of the light-cone. The vector generating these diffeomorphisms is

$$
\begin{array}{r}
\xi^{t}=f(\theta) \rho^{1 / 2}, \\
\xi^{\rho}=-\frac{2}{t} f(\theta) \rho^{3 / 2}, \\
\xi^{i}=\frac{1}{t} \rho^{3 / 2} g_{(0)}^{i j} \partial_{j} f(\theta) .
\end{array}
$$

The BMS transformations are known to be the transformations at null infinity of asymptotically Minkowski spacetime (with a suitable definition of the notion "asymptotically Minkowski.") It should be stressed that our conditions for the asymptotic metric (6.1) are more general than that of BMS and thus the asymptotic diffeomorphisms contain the BMS group as a subgroup. 


\section{References}

[1] G. 't Hooft, "Dimensional reduction in Quantum Gravity", in Salamfestschrift: A Collection of Talks, World Scientific Series in 20th Century Physics, Vol. 4, ed. A. Ali, J. Ellis and S. Randjbar-Daemi (World Scientific, 1993), gr-qc/9310026.

[2] L. Susskind, "The World as a Hologram", J. Math. Phys. 36 (1995) 6377, hep-th/9409089.

[3] J. Maldacena, "The Large N Limit of Superconformal Field Theories and Supergravity", Adv. Theor. Math. Phys. 2 (1998) 231, hep-th/9711200.

[4] S. Gubser, I. Klebanov and A. Polyakov, "Gauge Theory Correlators from Non-Critical String Theory", Phys. Lett. B428 (1998) 105, hep-th/9802109.

[5] E. Witten, "Anti De Sitter Space And Holography", Adv. Theor. Math. Phys. 2 (1998) 253, hep-th/9802150.

[6] E. Witten, "Quantum Gravity In De Sitter Space", hep-th/0106109.

[7] A. Strominger, "The dS/CFT Correspondence", hep-th/0106113.

[8] R. Bousso, "A Covariant Entropy Conjecture," JHEP 9907, 004 (1999) arXiv:hep-th/9905177.

[9] J. Polchinski, "S-matrices from AdS spacetime," arXiv:hep-th/9901076.

[10] J. D. Brown and M. Henneaux, "Central charges in the canonical realization of asymptotic symmetries: an example from three dimensional gravity", Commun. Math. Phys. 104 (1986) 207.

[11] H. Bondi, M. G. van der Burg and A. W. Metner, Waves From Axisymmetric Isolated Systems," Proc. Roy. Soc. Lond. A 269 (1962) 21.

[12] P. Sommers, J. Math. Phys. 19 (1978), 549.

[13] A. Ashtekar, "Asymptotic structure of the gravitational field at spatial infinity", In: General Relativity and Gravitation, ed. A. Held (Plenum Press, NY, 1980).

[14] S. W. Hawking and G. F. R. Ellis, The Large Scale Structure of Space-Time (Cambridge: Cambridge University Press).

[15] C. Fefferman and C. R. Graham: "Conformal Invariants". In: Elie Cartan et les Mathematiques d'aujord'hui, (Asterisque, 1985), 95.

[16] I. M. Gel'fand and G. E. Shilov, Generalized functions, vol. I (Academic Press, NY, 1964).

[17] V. Balasubramanian, J. de Boer and D. Minic, "Exploring de Sitter space and holography," arXiv:hep-th/0207245. 
[18] J. Bros, J.-P. Gazeau and U. Moschella, "Quantum Field Theory In The De Sitter Universe," Phys. Rev. Lett. 73 (1994) 1746; J. Bros and U. Moschella, Rev. Math. Phys. 8, 327 (1996) arXiv:gr-qc/9511019; J. Bros, H. Epstein and U. Moschella, Commun. Math. Phys. 196, 535 (1998) arXiv:gr-qc/9801099; M. Bertola, J. Bros, V. Gorini, U. Moschella and R. Schaeffer, Nucl. Phys. B 581, 575 (2000) arXiv:hep-th/0003098.

[19] M. Bertola, V. Gorini, U. Moschella and R. Schaeffer, Phys. Lett. B 462, 249 (1999) arXiv:hep-th/9906035.

[20] S. de Haro, K. Skenderis and S. N. Solodukhin, "Gravity in warped compactifications and the holographic stress tensor," Class. Quant. Grav. 18, 3171 (2001) arXiv:hep-th/0011230.

[21] I. S. Gradshteyn and I. M. Ryzhik, Tables of Integrals, Series and Products (New York: Academic Press).

[22] R. Bousso, A. Maloney and A. Strominger, Phys. Rev. D 65, 104039 (2002) arXiv:hep-th/0112218.

[23] M. Spradlin and A. Volovich, "Vacuum states and the S-matrix in dS/CFT," Phys. Rev. D 65, 104037 (2002) arXiv:hep-th/0112223.

[24] V. Balasubramanian, S. B. Giddings and A. E. Lawrence, JHEP 9903, 001 (1999) arXiv:hep-th/9902052.

[25] S. B. Giddings, Phys. Rev. Lett. 83, 2707 (1999) arXiv:hep-th/9903048.

[26] J. D. Bjorken and S. D. Drell, Relativistic Quantum fields, vol.I (McGRaw-Hill, Inc.).

[27] M. Henningson and K. Skenderis, "The holographic Weyl anomaly", JHEP 9807 (1998), 023, hep-th/9806087; "Holography and the Weyl anomaly", hep-th/9812023.

[28] C. Imbimbo, A. Schwimmer, S. Theisen and S. Yankielowicz, "Diffeomorphisms and Holographic Anomalies", Class. Quant. Grav. 17 (1999) 1129, hep-th/9910267.

[29] F. Oberhettinger, Tables of Mellin Transforms, Springer Verlag, New York, 1974.

[30] S. N. Solodukhin, "How to make the gravitational action on non-compact space finite", Phys.Rev. D62 (2000), 044016, hep-th/9909197.

[31] R. Beig and B.G. Schmidt, "Einstein's equations near Spatial Infinity", Commun.Math.Phys. 87 (1982) 65-80.

[32] R. Beig, "Integration of Einstein's equations near spatial infinity", Proc.R.Soc.Lond. bf A 391 (1984) 295-304.

[33] K. Skenderis and S. N. Solodukhin, "Quantum effective action from the AdS/CFT correspondence", Phys. Lett. B432 (2000) 316-322, hep-th/9910023.

[34] J.D. Brown and J.W. York, "Quasilocal energy and conserved charges derived from the gravitational action", Phys.Rev. D47 (1993), 1407-1419. 